\title{
Disease dynamics of Honeybees with Varroa destructor as parasite and virus vector
}

\author{
Yun Kang ${ }^{1}$, Krystal Blanco ${ }^{2}$, Talia Davis ${ }^{3}$, Ying Wang ${ }^{4}$ and Gloria DeGrandi-Hoffman ${ }^{5}$
}

\begin{abstract}
The worldwide decline in honeybee colonies during the past 50 years has often been linked to the spread of the parasitic mite Varroa destructor and its interaction with certain honeybee viruses carried by Varroa mites. In this article, we propose a honeybee-mite-virus model that incorporates (1) parasitic interactions between honeybees and the Varroa mites; (2) five virus transmission terms between honeybees and mites at different stages of Varroa mites: from honeybees to honeybees, from adult honeybees to the phoretic mites, from brood to the reproductive mites, from the reproductive mites to brood, and from adult honeybees to the phoretic mites; and (3) Allee effects in the honeybee population generated by its internal organization such as division of labor. We provide completed local and global analysis for the full system and its subsystems. Our analytical and numerical results allow us have a better understanding of the synergistic effects of parasitism and virus infections on honeybee population dynamics and its persistence. Interesting findings from our work include: (a) Due to Allee effects experienced by the honeybee population, initial conditions are essential for the survival of the colony. (b) Low adult honeybees to brood ratios have destabilizing effects on the system which generate fluctuating dynamics that lead to a catastrophic event where both honeybees and mites suddenly become extinct. This catastrophic event could be potentially linked to Colony Collapse Disorder (CCD) of honeybee colonies. (c) Virus infections may have stabilizing effects on the system, and parasitic mites could make disease more persistent. Our model illustrates how the synergy between the parasitic mites and virus infections consequently generates rich dynamics including multiple attractors where all species can coexist or go extinct depending on initial conditions. Our findings may provide important insights on honeybee viruses and parasites and how to best control them.
\end{abstract}

Keywords: Allee Effects; Honeybees; Extinction; Virus; Parasite; Colony Collapse Disorder (CCD)

\section{Introduction}

Honeybees are the world's most important pollinators of food crops. It is estimated that one third of food that we consume each day mainly relies on pollination by bees. For example, in the United States, honeybees are major pollinators of alfalfa, apples, broccoli, carrots and many other crops, and hence are of economic importance. Honeybees have an estimated monetary value between $\$ 15$ and $\$ 20$ billion dollars annually as commercial pollinators in the U.S [29]. There are growing concerns both locally and globally that despite a $50 \%$ growth in honeybee stocks, the supply cannot keep up with the over $300 \%$ increase in agricultural demands [75]. Therefore, the recent sharp declines in honeybee populations have been considered as a global crisis. The most recent data from the 2012-2013 winter has shown an average loss of $44.8 \%$ of hives in the

\footnotetext{
${ }^{1}$ Sciences and Mathematics Faculty, College of Letters and Sciences, Arizona State University, Mesa, AZ 85212, USA (yun.kang@asu.edu)

${ }^{2}$ Simon A. Levin Mathematical and Computational Modeling Sciences Center, Arizona State University, Tempe, AZ 85281, USA

${ }^{3}$ School of Mathematical and Statistical Sciences, Arizona State University, Tempe, AZ 85281, USA

${ }^{4}$ School of Life Sciences, Arizona State University, Tempe, AZ 85281, USA,

${ }^{5}$ Carl Hayden Bee Research Center, USDA-ARS, 2000 East Allen Road, Tucson, AZ 85719.
}

Preprint submitted to Elsevier

February 22, 2016

(C) 2016. This manuscript version is made available under the Elsevier user license http://www.elsevier.com/open-access/userlicense/1.0/ 
U.S., and a total of $30.6 \%$ loss of commercial hives [65]. Some beekeepers have reported a loss of $90 \%$ of their hives $[20,46]$.

Between 1972 and 2006, the wild honeybee populations declined severely and are now considered virtually nonexistent $[44,73]$. Hence the use of commercial honeybees for pollination is extremely important. Beginning in 2006, beekeepers began to report an unusual phenomenon in dying bee colonies. Worker bees would leave the colony to forage and never return, leaving the queen and the young behind to die. No dead worker bees were found at the nest sites; they simply disappear [13, 68]. This phenomenon is known as Colony Collapse Disorder (CCD), which is a serious problem threatening the health of honeybees and therefore the economic stability of commercial beekeeping and pollination operations.

The exact causes and triggering factors for CCD have not been completely understood yet. Researchers have proposed several possible causes of CCD including stress on nutritional diet, harsh winter conditions, lack of genetic diversity, exposure to certain pesticides, diseases, and parasitic mites Varroa destructor which are also vectors of viral diseases of honeybees $[29,52]$. Even before CCD was detected in honeybee colonies, studies showed that most of the loses could be generally attributed to two main causes: the vampire mite, Varroa destructor, which feeds on host haemolymph, weakens host immunity and exposes the bees to a variety of viruses, and the tracheal mite, which infests the breathing tubes of the bee, punctures the tracheal wall and sucks the bee's blood and also exposes the bee to a variety of viruses [57,63, 41]. Since then, Varroa mites have been implicated as the main culprit in dying colonies. For example, in Canada, Varroa mites have been found to be the main reason behind wintering losses of bee colonies [25], and more generally studies have shown that if the mite population is not properly controlled, the honeybee colony will die [62]. Recent studies also suggest that the Varroa mite could be a contributing cause of CCD since they not only ectoparasitically feed on bees, but also vertically transmit a number of deadly viruses to the bees [37, 35]. There have been at least 14 viruses found in honeybee colonies $[4,37]$, which can differ in intensity of impact, virulence, etc. for their host. For example, the Acute Bee Paralysis Virus (ABPV) affects the larvae and pupae which fail to metamorphose to adult stage, while in contrast the Deformed Wing Virus (DWV) affects larvae and pupae, which can still survive to the adult stage [69].

Mathematical models are powerful tools that can provide insights on potential ecological processes that link to CCD with other factors that contribute to colony mortality. There are several models of honeybee colony population dynamics that include the effects of Varroa mites (see refs. in Becher et al. [6]). DeGrandi-Hoffman et al. [16] produced the first time-based honeybee colony growth model. Later, the population dynamics of Varroa mites were added [15]. In this model, the impact of mites on colony population growth is based on reductions in adult worker longevity following parasitism during immature stages. Martin [40] developed a simulation model consisting of ten components, that connected various aspects of honeybee and mite biology and later included effects of viral pathogens to explain the link between the Varroa mite and the collapse of colonies [41]. Wilkinson and Smith [74] developed a difference equation model of Varroa mites reproducing in a honeybee colony. Their study focused on parameter estimations and sensitivity analysis. Simulation models are useful but may be too complex to study mathematically and obtain general predictions.

More recently, mathematical models have been formulated to explore potential mechanisms causing CCD in honeybees. Eberl et al. [18] developed a model connecting Varroa mites to CCD by including brood maintenance terms. They found an important threshold for the number of hive worker bees needed to maintain and care of the brood. Khoury et al. $[32,31]$ developed differential equations models to study different death rates of foragers and the impact it had on colony growth and development. They then linked their results to CCD. Martin [41] constructed a Varroa population model that included mortality from virus and later modeled the effects of a constant population of Varroa mites on the brood and on adult worker bees, and found that sufficiently large mite infestations may make hives vulnerable to collapse from viral epidemics [66]. Betti et al. [8] constructed a model that combines the dynamics of the spread of disease within a bee colony with the underlying demographic dynamics of the colony to determine its ultimate fate under 
different scenarios. Their results suggest that the age of recruitment of hive bees to foraging duties is a good early marker for the survival or collapse of a honeybee colony in the face of infection. Kribs-Zaleta et al. [34] created a model to account for both healthy hive dynamics and hive extinction due to CCD by modeling CCD via a transmissible infection brought into the hive by foragers. Perry et al. [50] examined the social dynamics underlying dramatic colony failure. Their model includes bee foraging performance varying with age, and displays dynamics of colony population collapse that are similar to field reports of CCD. These models, no doubt, are insightful and provide us a better understanding on the potential mechanisms that link to CCD. However, most of these models only account for the honeybee population dynamics with mites or viruses but not both.

The host-parasite relationship between honeybees and Varroa mites is complicated by the mite's close association with a wide range of honeybee viral pathogens. In order to understand how Varroa mite infestations and the related viruses transmitted to honeybees affect honeybee population dynamics, and which may link to CCD, there is a need to develop realistic and mathematically tractable models that include both virus and mite population dynamics. The goal of our work is to develop a useful honeybee-mite-virus system to obtain a better understanding on the synergistic effects of honeybee-mite interactions and honeybee-virus interactions on the honeybee population dynamics, thus develop good practices to control these parasites to maintain or increase honeybee populations. The most relevant modeling papers for our study purposes are by Sumpter and Martin [66], and Ratti et al. [52] whose work examined the transmission of viruses via Varroa mites, using the susceptible-infectious (SI) disease modeling framework with mites as vectors for transmission. However, Sumpter and Martin assumed that the mites' population is constant while Ratti et al. took no account of the fact that virus transmissions occur at different biological stages of Varroa mites and honeybees.

In this article, we follow both approaches of Sumpter and Martin [66] and Ratti et al. [52], and propose a honeybee-mite-virus model that incorporates (1) parasitic interactions between honeybees and Varroa mites; (2) different virus transmission terms that account for the virus transmissions among honeybees, between honeybees and mites at two different stages of Varroa mites; and (3) Allee effects in the honeybee population generated by the internal organization of honeybees (e.g., division of labor). Our proposed model will allow us explore the following questions:

1. What are the dynamics of a system only consisting of honeybees and the virus?

2. What are the dynamics of a system only consisting of honeybees and Varroa mites?

3. What are the synergistic effects of Varroa mites and the virus on the honeybee population, and how may these synergistic effects contribute to CCD?

4. How can we maintain honeybee populations?

The structure of the remainder of the article is organized as follows: In Section 2, we first provide the biological background of honeybees, Varroa mites, and the associated virus transmission routes in the honeybee-mite system; then we derive our SI-type model for honeybees co-infected with the mite and virus. In Section 3, we perform local and global analysis of the proposed model and the related subsystems. The results from the analysis are then connected to biological contexts and implications. Additionally, we also explore numerical simulations of the subsystems and the full system to obtain the effects of each parameter in our system. In Section 4, we summarize our results and the related biological implications of our studies in finding potential causes of Colony Collapse Disorder. We also provide potential projects for future work. The detailed mathematical proofs of our theoretical results are provided in the last section. 


\section{Biological background and model derivations}

Honeybee colony: During the spring and summer, a honeybee colony typically consists of a single reproductive queen, 20,000 - 60,000 adult worker bees, 10,000 - 30,000 individuals at the brood stage (egg, larvae and pupae) and up to hundreds of male drones. During the winter, the colony typically reduces in size and consists of a single queen and somewhere between 8,000-15,000 worker bees [41]. A large population of workers carry out the tasks of the bee colony, which include foraging, pollination, honey production and, in particular, caring for the brood and rearing the next generation of bees. The queen is the only fertile individual of the colony and has an average life span of $2-3$ years [66]. During the peak season (in the summer), the queen lays up to 2000 eggs per day, where fertilized eggs produce female worker bees, or much more rarely queens, while drones develop from non-fertilized eggs [8]. The bees go through the following stages in development: egg (about 3 days), larvae (about 7 days), pupae (about 14 days), and adult. The life span of an adult worker bee also depends on the season. Workers usually have a lifespan of $3-6$ weeks during the spring and summer, and are reported to live as long as 4 months during the winter [47]. The adult drone life span is typically 20 - 40 days, with reports of drone living up to 59 days under optimal colony conditions $[47,27]$.

Let $N_{h}(t)$ be the total number of honeybees in the colony, including the larvae, pupae and adult bees (both hives and foragers) at time $t$. The subscript $h$ means honeybee for all future notations. Define $\xi_{h} \in[0,1]$ as the proportion of adult honeybee population in the colony, then $\left(1-\xi_{h}\right) N_{h}$ is the brood population and $\frac{\xi_{h}}{1-\xi_{h}}$ is the ratio of adult honeybees to brood in colony. Empirical study shows that the successful honeybee colony should have $\frac{\xi_{h}}{1-\xi_{h}}>2$ (see [59]). We should expect that the value of $\xi_{h}$ varies with time. In our current model, instead of employing the explicit age structure model, we let $\xi_{h}$ be a parameter. Though brood and adult numbers change throughout the year, the ratio $\xi_{h}$ remains at the steady state of the explicit age structure model which serves as a limiting factor in proportion of eggs that are reared into larvae and emerge as adults. Under this simplification, we are able to obtain analytical results on how $\xi_{h}$ affects dynamics of the model with essential biological components. In the absence of mites and virus, the population dynamics of honeybees $N_{h}(t)$ is described by the following nonlinear equation:

$$
N_{h}^{\prime}=\frac{r\left(\xi_{h} N_{h}\right)^{2}}{K+\left(\xi_{h} N_{h}\right)^{2}}-d_{h} N_{h}
$$

where' ${ }^{\prime}$ is the sign of the derivative with respect to time; the parameter $r$ is specified as the average number of eggs laid by the queen per day; the parameter $\sqrt{K}$ is the size of the bee colony at which the egg laying rate is half of $r$; and $d_{h}$ is the average death rate of the workers. The term $\frac{\left(\xi_{h} N_{h}\right)^{2}}{K+\left(\xi_{h} N_{h}\right)^{2}}$ describes that the successful survival of an egg which will develop into a worker bee needs the care of adult workers $\left(\xi_{h} N_{h}\right)$ inside the colony and also needs food brought in by foragers. This term also implicitly assumes that more adult workers inside the colony can increase the survival of an egg and the development into an adult that is supported by empirical evidence reported in [59]. Our modeling approach is similar to Eberl et al [18] for honeybee diseases and Kang et al [30] for the population dynamics of leaf-cutter ants. This assumption includes the internal organization of the colony, division of labor. Model (1) implies that the colony is able to survive when $\xi_{h}$ is above a threshold, i.e., $\xi_{h}>\frac{2 d_{h} \sqrt{K}}{r}$. More detailed analysis and related results are provided in the next section.

Varroa mites: Varroa mites were first reported in Kentucky in the Bluegrass region of the Commonwealth in 1991 in U.S. They have since spread to become a major pest of honeybees in many states of U.S. [7]. Varroa mites are external honeybee parasites that attack both adult honeybees and brood, with a distinct preference for drone brood [48]. They suck the blood from both adult workers and the developing brood, weakening them and shortening the life span of the bees which they feed on. Emerging brood may be born with deformed wings. Untreated infestations of Varroa mites can cause honeybee colonies to collapse [39]. 

(all female) undergo two phases in their life cycle, the phoretic and reproductive phases. During the phoretic phase, Varroa feed on adult bees and are passed from bee to bee as they pass one another in the colony. During the phoretic phase, the mites live on adult bees and can usually be found between the abdominal segments of the bees. The mites puncture the soft tissue between the segments and feed on bee hemolymph, harming the host [54, 9]. Mite reproduction can occur only if brood is available. A mature female mite enters the brood cell about one day before capping and will be sealed in with the larva. After the capping of the cell, it lays a single male egg and several female eggs at 30-hour intervals [70], and the mite feeds and develops on the maturing bee larva. When the host bee leaves the cell, the mature female mites leave the cell. The male mite dies after mating with his sisters, and if immature female mites are present they die as they come out of the cell, as they cannot survive once outside the cell. The adult female mite begins searching for other bees or larvae to parasitize.

The phoretic period of the mite appears to contribute to the mite's reproductive ability, which may last 4.5 to 11 days when brood is present in the hive; or as long as five to six months during the winter when little or no brood is present in the hive. Consequently, mites living when brood is present in the colony have an average life expectancy of 27 days, yet in the absence of brood, they may live for many months. In the average temperate climate, mite populations can increase 12 -fold in colonies which have brood half of the year and 800-fold in colonies which have brood year-round. This period usually begins in late winter when brood rearing resumes from a winter period when little or no brood is present. The period of mite population increase continues through the spring and summer and peaks in the fall when brood rearing is nearly done. This makes the mites very difficult to control, especially in warmer climates where colonies maintain brood year-round [21].

Let $N_{m}(t)$ be the number of adult mites in the honeybee colony in the absence of a virus where the subscript $m$ means mite for all future notations. Varroa mites feed on the haemolymph of brood and adult honeybees, and their reproduction depends on the availability of the brood and the population of the reproductive mites. Similarly to the case of honeybees, we incorporate an implicit age structure model of Varroa mites by defining a parameter $\xi_{m} \in[0,1]$ as the proportion of mites at the phoretic stage. This implies that the reproductive mite population has size of $\left(1-\xi_{m}\right) N_{m}$ and the phoretic mite population is of size $\xi_{m} N_{m}$. This simplification still allows us to investigate the parasitic interactions between mites and honeybees rigorously with essential biological components.

We model the parasitic interactions between mites and honeybees by using the Holling Type I functional responses, i.e. $\hat{\alpha}\left(1-\xi_{h}\right) N_{h}\left(1-\xi_{m}\right) N_{m}=\alpha N_{h} N_{m}$ where $\hat{\alpha}$ is the parasitism rate; the term $\left(1-\xi_{h}\right) N_{h}$ is the brood population; the term $\left(1-\xi_{m}\right) N_{m}$ is the reproductive mites population; and let $\alpha=\hat{\alpha}\left(1-\xi_{h}\right)\left(1-\xi_{m}\right)$. Therefore, in the presence of mites $N_{m}$, the dynamics of the honeybee population $N_{h}$ can be described as:

$$
N_{h}^{\prime}=\frac{r\left(\xi_{h} N_{h}\right)^{2}}{K+\left(\xi_{h} N_{h}\right)^{2}}-d_{h} N_{h}-\alpha N_{h} N_{m}=\frac{r\left(\xi_{h} N_{h}\right)^{2}}{K+\left(\xi_{h} N_{h}\right)^{2}}-\left(d_{h}+\alpha N_{m}\right) N_{h}
$$

which implies that the parasitism decreases the life span of the honeybee, i.e., the average life span has been reduced to $\frac{1}{d_{h}+\alpha \mathbf{N}_{m}}$ after parasitism. Here we do not assume that parasitism would lead to certain death of the honeybees. The Varroa mite population depends on the nutrient obtained from honeybees, thus, the dynamics of mites population can be described as:

$$
N_{m}^{\prime}=c \alpha N_{h} N_{m}-d_{m} N_{m}
$$

where the parameter $\alpha$ measures the parasitic rate of Varroa mites; $c$ is the conversion rate from nutrient consumption obtained from honeybees to sustenance for Varroa mites reproduction; and $d_{m}$ is the natural death rate of Varroa mites. Therefore, in the absence of virus, the population dynamics of mites and 


$$
\begin{aligned}
N_{h}^{\prime} & =\frac{r\left(\xi_{h} N_{h}\right)^{2}}{K+\left(\xi_{h} N_{h}\right)^{2}}-d_{h} N_{h}-\alpha N_{h} N_{m} \\
N_{m}^{\prime} & =c \alpha N_{h} N_{m}-d_{m} N_{m}
\end{aligned}
$$

The modeling approach of Model (2) has applied the traditional host-parasite modeling framework including non-lethal parasites [1,2]. Model (2) also implies that Varroa mites population $N_{m}$ goes extinct if the population of honeybees $N_{h}$ goes extinct.

Varroa mites act as a virus-vector for virus transmissions: Varroa mites reproduce in cells with developing worker or drone larvae. Just before a brood cell is sealed, a female mite (i.e., foundress) will invade and parasitize the larvae thus beginning the reproductive phase (for details see Rosenkranz et al. [54]). The offspring (i.e., daughter mites) will mate in the cells. When the adult bee emerges, the foundress mother mite and her daughters will leave the cell. The mites will attach to adult bees (phoretic phase) until they find a new cell where they can invade and reproduce. The mites can feed on the adult bee during the phoretic phase. To model the virus transmissions between mites and honeybees during these two phases, we let $S_{h}(t), S_{m}(t)$ be the susceptible population of honeybees and mites, respectively; and $I_{h}(t), I_{m}(t)$ be the virus infected population of honeybees and mites, respectively. Then the total population of honeybees is $N_{h}(t)=S_{h}(t)+I_{h}(t)$, and the total population of mites is $N_{m}(t)=S_{m}(t)+I_{m}(t)$.

The virus transmissions between female mites and honeybees can occur in the following two phases of the mite life cycle:

1. The honeybee colony has $\xi_{h} S_{h}$ susceptible adult honeybees; $\xi_{h} I_{h}$ virus infected adult honeybees, $\xi_{m} S_{m}$ susceptible phoretic mites; and $\xi_{m} I_{m}$ virus infected phoretic mites. In the phoretic phase, mites move between adult bees both spontaneously and just prior to the death of bee [66]. Following the approach of [41], we assume that virus transmissions are frequency dependent, i.e.,

- We model the rate at which the susceptible adult honeybees are virus infected by infected phoretic mites (IPFM) based on the approach of $[41,66,18,52]$. This rate can be described as follows:

$$
\begin{aligned}
& \underbrace{\beta_{m h}}_{\text {probability being infected after contacts }} \times \underbrace{\xi_{m} I_{m}}_{\text {population of infected IPFM }} \times \underbrace{\frac{\xi_{h} S_{h}}{\xi_{h} S_{h}+\xi_{h} I_{h}}}_{\text {probability contacts susceptible adult honeybees }} \\
& =\frac{\beta_{m h} \xi_{m} S_{h} I_{m}}{S_{h}+I_{h}} .
\end{aligned}
$$

which also implies that susceptible honey bees become virus infected at a rate proportional to the ratio of the infected phoretic mite population to the total honeybee population.

- The rate at which susceptible phoretic mites (SPFM) are virus infected by virus infected adult honeybees (IAH) is:

$$
\begin{aligned}
& \quad \underbrace{\beta_{h m}}_{\text {probability being infected after contacts }} \times \underbrace{\xi_{m} S_{m}}_{\text {population of SPFM }} \times \underbrace{\frac{\xi_{h} I_{h}}{\xi_{h} S_{h}+\xi_{h} I_{h}}}_{\text {probability being contacted by IAH }} \\
& =\frac{\beta_{h m} \xi_{m} S_{m} I_{h}}{S_{h}+I_{h}} .
\end{aligned}
$$

2. The honeybee colony has $\left(1-\xi_{h}\right) S_{h}$ susceptible brood; $\left(1-\xi_{h}\right) I_{h}$ virus infected brood, $\left(1-\xi_{m}\right) S_{m}$ susceptible reproductive mites; and $\left(1-\xi_{m}\right) I_{m}$ virus infected reproductive mites. We do not assume that brood will die from parasitism, thus, parasitised brood or daughter mites will face virus infection 
if either brood or the mother mite is virus infected. Chen et al. found a direct relationship between virus frequency and the number of mites to which brood were exposed, i.e., the more donor mites that were introduced per cell, the greater the incidence of virus that was detected in brood [11, 12]. This implies that the virus transmission rate between mites and brood during the reproductive phase of mites is density dependent, i.e., similar to the term that describes the parasitic interaction between mites and honeybee. Therefore, we have follows:

- A developing bee becomes virus-infected if it is parasitized by an infected foundress mite. Thus, the rate at which the susceptible brood is infected by virus infected reproductive mites (IRFM), i.e., infected foundress mites, is:

$$
\begin{aligned}
& \qquad \underbrace{\beta_{m h 2}}_{\text {probability being infected after contacts }} \times \underbrace{\left(1-\xi_{h}\right) S_{h}}_{\text {population of healthy honeybee brood }} \times \underbrace{\hat{\alpha}\left(1-\xi_{m}\right) I_{m}}_{\text {parasitism by IRFM }} \\
& =\beta_{m h 2} \alpha S_{h} I_{m} .
\end{aligned}
$$

- The reproduction of mites depends on brood. Daughter mites can become virus infected if either brood or their mother is virus infected. Thus, based on the formulation of the host-parasite interaction model (2), the rate at which daughter mites (INFM) become virus infected depends on the parasitic interaction between mites and honeybees described as $c \alpha\left[I_{h}\left(S_{m}+I_{m}\right)+S_{h} I_{m}\right]$ where the term $I_{h}\left(S_{m}+I_{m}\right)$ is the daughter mites infected with virus infected brood; and the term $S_{h} I_{m}$ is the daughter mites infected with virus via their mothers.

The virus transmission among honeybees: The proportion of honeybees which can infect themselves is also dependent on the total number of susceptible and virus infected bees present in the colony, and hence frequency-dependent transmission is used [41], which is described as follows:

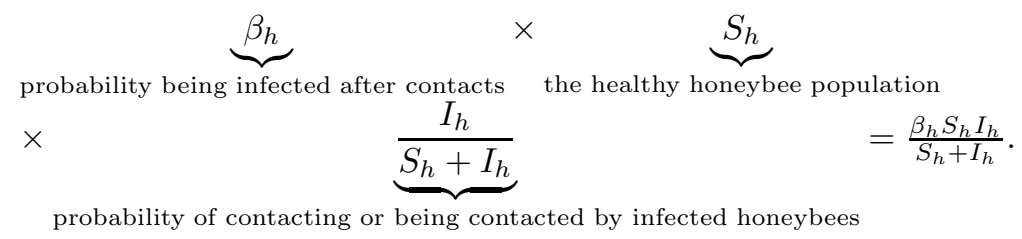

The reduced fitness of honeybees due to virus infections: The virus infections contribute to morphological deformities of honeybees such as small body size, shortened abdomen and deformed wings, which reduce vigor and longevity, and they can also influence flight duration and the homing ability of foragers [35]. To include the effects of virus infections in our model, we assume that the virus infected adult honeybee population $\xi_{h} I_{h}$ affects brood rearing rates and colony population growth with a reduced fitness measured by $\rho \in(0,1)$. The healthy honeybee population $S_{h}$ (i.e., un-infected honeybees) can be modeled as follows,

$$
\begin{aligned}
S_{h}^{\prime}= & \underbrace{\frac{r \xi_{h}^{2}\left(S_{h}+\rho I_{h}\right)^{2}}{K+\xi_{h}^{2}\left(S_{h}+\rho I_{h}\right)^{2}}}_{\text {reproduction of honeybees }}-\underbrace{\frac{\beta_{h} S_{h} I_{h}}{S_{h}+I_{h}}}_{\text {honeybee infected by nestmates }}-\underbrace{\alpha S_{h}\left(S_{m}+I_{m}\right)}_{\text {parasitism by mites }} \\
& -\underbrace{\frac{\beta_{m h}\left(\xi_{m} S_{h}\right) I_{m}}{S_{h}+I_{h}}}_{\text {adult honeybees infected by the phoretic mites }}-\underbrace{\beta_{m h 2} \alpha S_{h} I_{m}}_{\text {brood infected by the reproductive mites }}-d_{h} S_{h}
\end{aligned}
$$

And the virus infected honeybee population can be modeled by the following equation, 


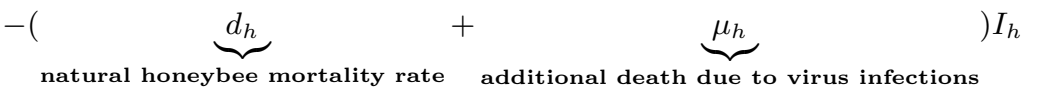

Let $\mu_{m}$ be the additional death rate of mites due to virus infections. In the case that virus infections do not cause additional mortality of mites, the value of $\mu_{m}$ is zero. The population of healthy mites $S_{m}$ and the virus infected mites $I_{m}$ can be described by the following set of nonlinear equations:

$$
\begin{aligned}
& S_{m}^{\prime}=S_{m}\left[\begin{array}{c}
c \alpha S_{h}-\underbrace{\frac{\beta_{h m} \xi_{m} I_{h}}{S_{h}+I_{h}}}_{\text {the phoretic mites infected by adult honeybees }}-\underbrace{d_{m}}_{\text {natural mortality rate of mites }}
\end{array}\right] \\
& I_{m}^{\prime}=c \alpha \underbrace{\left[I_{h}\left(S_{m}+I_{m}\right)+S_{h} I_{m}\right]}_{\text {mites born with virus infections }}+\underbrace{\mu_{m}}_{\text {additional death due to virus infections }}) I_{m}
\end{aligned}
$$

Based on the above, the full model of honeybee-mites-virus population dynamics is modeled by the following system of differential equations:

$$
\begin{aligned}
S_{h}^{\prime}= & \frac{r \xi_{h}^{2}\left(S_{h}+\rho I_{h}\right)^{2}}{K+\xi_{h}^{2}\left(S_{h}+\rho I_{h}\right)^{2}}-d_{h} S_{h}-\frac{\beta_{h} S_{h} I_{h}}{S_{h}+I_{h}}-\frac{\beta_{m h}\left(\xi_{m} S_{h}\right) I_{m}}{S_{h}+I_{h}} \\
& -\beta_{m h 2} \alpha I_{m}-\alpha S_{h}\left(S_{m}+I_{m}\right) \\
I_{h}^{\prime}= & S_{h}\left[\frac{\beta_{h} I_{h}}{S_{h}+I_{h}}+\frac{\beta_{m h} \xi_{m} I_{m}}{S_{h}+I_{h}}+\beta_{m h 2} \alpha I_{m}\right]-\alpha I_{h}\left(S_{m}+I_{m}\right)-\left(d_{h}+\mu_{h}\right) I_{h} . \\
S_{m}^{\prime}= & S_{m}\left[c \alpha S_{h}-\frac{\beta_{h m} \xi_{m} I_{h}}{S_{h}+I_{h}}-d_{m}\right] \\
I_{m}^{\prime}= & c \alpha\left[I_{h}\left(S_{m}+I_{m}\right)+S_{h} I_{m}\right]+\frac{\beta_{h m} I_{h}\left(\xi_{m} S_{m}\right)}{S_{h}+I_{h}}-\left(d_{m}+\mu_{m}\right) I_{m}
\end{aligned}
$$

For convenience, let $\hat{K}=\frac{K}{\xi_{h}^{2}}, \hat{\beta}_{m h}=\beta_{m h} \xi_{m}, \tilde{\beta}_{m h}=\beta_{m h 2} \alpha, \hat{\beta}_{h m}=\beta_{h m} \xi_{m}$. Then the full model (6) can be rewritten as the following model

$$
\begin{aligned}
S_{h}^{\prime} & =\frac{r\left(S_{h}+\rho I_{h}\right)^{2}}{\hat{K}+\left(S_{h}+\rho I_{h}\right)^{2}}-d_{h} S_{h}-\frac{\beta_{h} S_{h} I_{h}}{S_{h}+I_{h}}-\frac{\hat{\beta}_{m h} S_{h} I_{m}}{S_{h}+I_{h}}-\tilde{\beta}_{m h} S_{h} I_{m}-\alpha S_{h}\left(S_{m}+I_{m}\right) \\
I_{h}^{\prime} & =S_{h}\left[\frac{\beta_{h} I_{h}}{S_{h}+I_{h}}+\frac{\hat{\beta}_{m h} I_{m}}{S_{h}+I_{h}}+\tilde{\beta}_{m h} I_{m}\right]-\alpha I_{h}\left(S_{m}+I_{m}\right)-\left(d_{h}+\mu_{h}\right) I_{h} \\
S_{m}^{\prime} & =S_{m}\left[c \alpha S_{h}-\frac{\hat{\beta}_{h m} I_{h}}{S_{h}+I_{h}}-d_{m}\right] \\
I_{m}^{\prime} & =c \alpha\left[I_{h}\left(S_{m}+I_{m}\right)+S_{h} I_{m}\right]+\frac{\hat{\beta}_{h m} I_{h} S_{m}}{S_{h}+I_{h}}-\left(d_{m}+\mu_{m}\right) I_{m}
\end{aligned}
$$

where $\alpha>0, c>0, \rho \in[0,1]$ and the virus transmission rates $\beta_{h}, \hat{\beta}_{m h}, \hat{\beta}_{h m}, \tilde{\beta}_{m h} \in(0,1)$. In summary, the full honeybee-mite-virus model (7) incorporates (1) Allee effects of honeybees due to the cooperation of the internal organization; (2) parasitism interactions between honeybee and mites; (3) the vertical virus transmission mode modeled by the frequency-dependent virus transmission function during the mites' phoretic phase; (4) the horizontal virus transmission mode modeled by the density-dependent virus transmission func- 
tion during the mites' reproductive phase; and (5) the reduced fitness of honeybees due to virus infections. Model (7) allows us to investigate the following scenarios:

1. Population growth of colonies in the absence of mites and virus.

2. In the absence of the virus, how the Varroa mites may affect the population dynamics of colonies.

3. In the absence of the mites, how virus infections may affect the population dynamics of colonies. This case can apply to situations where honeybees are infected by virus through ecological processes such as foraging.

4. In the presence of both the mites and virus infections, which conditions can lead to the extinction of mites, virus infections, and colony population; and which conditions can guarantee colony survival.

The remainder of this manuscript will focus on the dynamics of Model (7) and the related subsystems.

\section{Mathematical analysis}

Let $\left.\frac{S_{h} I_{h}}{S_{h}+I_{h}}\right|_{S_{h}=I_{h}=0}=0$ and $\left.\frac{I_{m}}{S_{m}+I_{m}}\right|_{S_{m}=I_{m}=0}=0$. Define $X=\left\{\left(S_{h}, I_{h}, S_{m}, I_{m}\right) \in \mathbb{R}_{+}^{4}: S_{h}+I_{h}>\right.$ 0 and $\left.S_{m}+I_{m}>0\right\}$, then $X$ can be considered as the state space of our model (7). To continue the analysis, let us define $N_{h}=S_{h}+I_{h}, N_{m}=S_{m}+I_{m}$ and $N=c N_{h}+N_{m}$ as the population of honeybees, the population of mites, and the sum of the population of honeybees and mites, respectively. In addition, we let $d=\min \left\{d_{h}, d_{m}\right\}$, and define $N^{*}$ as the upper bound of the sum of the population of honeybees and mites and $N^{c}$ as the corresponding threshold, where

$$
N^{c}=c \frac{\frac{r}{d}-\sqrt{\left(\frac{r}{d}\right)^{2}-4 \hat{K}}}{2}, \quad N^{*}=c \frac{\frac{r}{d}+\sqrt{\left(\frac{r}{d}\right)^{2}-4 \hat{K}}}{2} .
$$

We let $\bar{N}_{h}^{*}, \underline{N}_{h}^{*}$ be the upper bound, lower bound of the population of honeybees, respectively, and $\bar{N}_{h}^{c}, \underline{N}_{h}^{c}$ be the corresponding thresholds, where

$$
\begin{array}{ll}
\bar{N}_{h}^{c}=\frac{\frac{r}{d_{h}}-\sqrt{\left(\frac{r}{d_{h}}\right)^{2}-4 \hat{K}}}{2}, & \bar{N}_{h}^{*}=\frac{\frac{r}{d_{h}}+\sqrt{\left(\frac{r}{d_{h}}\right)^{2}-4 \hat{K}}}{2} \\
\underline{N}_{h}^{c}=\frac{\frac{r}{d_{h}+\mu_{h}+\alpha N^{*}}-\sqrt{\left(\frac{r}{d_{h}+\mu_{h}+\alpha N^{*}}\right)^{2}-4 \hat{K} / \rho^{2}}}{2} & \underline{N}_{h}^{*}=\frac{\frac{r}{d_{h}+\mu_{h}+\alpha N^{*}}+\sqrt{\left(\frac{r}{d_{h}+\mu_{h}+\alpha N^{*}}\right)^{2}-4 \hat{K} / \rho^{2}}}{2} .
\end{array}
$$

And we let $S_{h}^{*}$ be the lower bound of the population of susceptible honeybees, and $S_{h}^{c}$ be the corresponding threshold, where

Define $f^{b}(x, y)=\frac{\frac{r}{x}+\sqrt{\left(\frac{r}{x}\right)^{2}-4 \hat{K} / y}}{2}$ and $f_{b}(x, y)=\frac{\frac{r}{x}-\sqrt{\left(\frac{r}{x}\right)^{2}-4 \hat{K} / y}}{2}$, then we have

$$
\frac{\partial f^{b}(x, y)}{\partial x}<0, \frac{\partial f^{b}(x, y)}{\partial y}<0, \frac{\partial f_{b}(x, y)}{\partial x}>0, \frac{\partial f_{b}(x, y)}{\partial y}>0
$$


which imply the following inequalities

$$
S_{h}^{c}<S_{h}^{c}<S_{h}^{*}<\bar{N}_{h}^{*}, N^{c} \leq \bar{N}_{h}^{c}<\underline{N}_{h}^{c}<\underline{N}_{h}^{*}<\bar{N}_{h}^{*} \leq N^{*} / c
$$

Theorem 3.1 (Basic dynamical properties). Assume that all parameters are strictly positive and $\rho \in$ $[0,1]$. The model $(7)$ is positively invariant and bounded in the state space $X$, which is attracted to the following compact set

$$
C=\left\{\left(S_{h}, I_{h}, S_{m}, I_{m}\right) \in \mathbb{R}_{+}^{4}: 0 \leq c\left(S_{h}+I_{h}\right)+\left(S_{m}+I_{m}\right)=c N_{h}+N_{m} \leq N^{*}\right\}
$$

provided that $\frac{r}{d}>2 \sqrt{\hat{K}}$ and time is large enough. Moreover, the following statements hold for Model (7):

- If $\frac{r}{2 \sqrt{\hat{K}}}>d_{h}$, then the total population of honeybees $N_{h}$ is bounded by $\bar{N}_{h}^{*}$, i.e.,

$$
\limsup _{t \rightarrow \infty} N_{h}(t) \leq \bar{N}_{h}^{*}
$$

If $\frac{r}{2 \sqrt{\hat{K}}}>\frac{d_{h}+\mu_{h}+\alpha N^{*}}{\rho}$ and $N_{h}(0)>\underline{N}_{h}^{c}$ hold, then the total population of honeybees $N_{h}$ is persistent,
i.e..

$$
\underline{N}_{h}^{*} \leq \liminf _{t \rightarrow \infty} N_{h}(t) \leq \limsup _{t \rightarrow \infty} N_{h}(t) \leq \bar{N}_{h}^{*} \leq \limsup _{t \rightarrow \infty} N(t) / c=N^{*} / c
$$

- If the inequalities $\frac{r}{2 \sqrt{\hat{K}}}>\max \left\{d_{h}+\beta_{h}+\frac{\hat{\beta}_{m h} N^{*}}{\underline{N}_{h}^{*}}+\left(\tilde{\beta}_{m h}+\alpha\right)\left(N^{*}-c \underline{N}_{h}^{*}\right), \frac{d_{h}+\mu_{h}+\alpha N^{*}}{\rho}\right\}$ with $N_{h}(0) \geq$ $S_{h}(0)>S_{h}^{c}$ hold, then $S_{h}$ is persistent with the following properties:

$$
S_{h}^{*} \leq \liminf _{t \rightarrow \infty} S_{h}(t) \leq \liminf _{t \rightarrow \infty} N_{h}(t) \leq \liminf _{t \rightarrow \infty} N(t) / c \leq \limsup _{t \rightarrow \infty} N(t) / c \leq N^{*} / c
$$

- The extinction equilibrium $E_{0}=(0,0,0,0)$ is always local stable. Moreover, the system (7) converges to $E_{0}$ globally if $d_{h}>\frac{r}{2 \sqrt{\hat{K}}}$ holds; and it converges to $E_{0}$ locally if the initial population satisfies either $N(0)<N^{c}$ or $N_{h}(0)<\bar{N}_{h}^{c}$.

Notes: The positive invariance and boundedness results from Theorem 3.1 imply that our model is welldefined biologically. In addition, the results of Theorem 3.1 indicate follows:

1. Initial conditions are important determinants of colony survival.

2. The inequality $\frac{r}{2 \sqrt{\hat{K}}}>d_{h}$ is a necessary condition for colony survival, i.e., the large intrinsic growth rate $r$, the small half saturation $\hat{K}$, and the small death rate of honeybees $d_{h}$.

3. Low rates of virus transmission and parasitism $\beta_{h}, \hat{\beta}_{m h}, \tilde{\beta}_{m h}, \alpha$ are also important for the persistence of the healthy honeybee population (i.e., uninfected) $S_{h}$.

Recall that $d=\min \left\{d_{h}, d_{m}\right\}, N^{c}=c \frac{\frac{r}{d}-\sqrt{\left(\frac{r}{d}\right)^{2}-4 \hat{K}}}{2}, N^{*}=c \frac{\frac{r}{d}+\sqrt{\left(\frac{r}{d}\right)^{2}-4 \hat{K}}}{2}$ and

$$
\underline{N}_{h}^{*}=\frac{\frac{r}{d_{h}+\mu_{h}+\alpha N^{*}}+\sqrt{\left(\frac{r}{d_{h}+\mu_{h}+\alpha N^{*}}\right)^{2}-4 \hat{K} / \rho^{2}}}{2} .
$$



$\underline{N}_{h}^{c}$ holds. Notice that $\xi_{h}$ is the ratio of adult honeybees in the colony, and one of sufficient conditions that guarantee colony survival is the following inequality:

$$
\frac{r}{2 \sqrt{\hat{K}}}=\frac{r \xi_{h}}{2 \sqrt{K}}>\frac{d_{h}+\mu_{h}+\alpha N^{*}}{\rho} \Leftrightarrow \frac{r \xi_{h}}{2 \sqrt{K}}-\frac{\alpha \sqrt{\left(\frac{r}{d}\right)^{2}-4 \frac{K}{\xi_{h}^{2}}}}{2 \rho}>\frac{d_{h}+\mu_{h}+\alpha \frac{r}{2 d}}{\rho} .
$$

Thus, Theorem 3.1 provides a critical function of the hives population $\xi_{h}$ such that colony will survive. In addition, notice that $\frac{r \xi_{h}}{2 \sqrt{K}}-\frac{\alpha \sqrt{\left(\frac{r}{d}\right)^{2}-4 \frac{K}{\xi_{h}^{2}}}}{2 \rho}$ is an increasing function of $\xi_{h}$, this implies that the larger adult workers to brood ratio $\xi_{h}$, the better colony growth and survival. This is supported by empirical evidence reported in [59]. In the following theorem, we provide theoretical results on the sufficient conditions that lead to the persistence and the extinction of virus population or the mites population.

Theorem 3.2 (Persistence and extinction of virus or mites). The following statements hold

- If $N^{*}<\frac{d_{m}}{\alpha}$, then the total population of mite $N_{m}$ goes extinct, i.e.,

$$
\limsup _{t \rightarrow \infty} N_{m}(t)=0
$$

where system (7) is attracted to the mite-free invariant set $M F=\left\{\left(S_{h}, I_{h}, S_{m}, I_{m}\right) \in \mathbb{R}_{+}^{4}: S_{m}+I_{m}=\right.$ $0\}$, and its dynamics is equivalent to the following two-D model (8)

$$
\begin{aligned}
S_{h}^{\prime} & =\frac{r\left(S_{h}+\rho I_{h}\right)^{2}}{\hat{K}+\left(S_{h}+\rho I_{h}\right)^{2}}-d_{h} S_{h}-S_{h} \frac{\beta_{h} I_{h}}{S_{h}+I_{h}} \\
I_{h}^{\prime} & =S_{h} \frac{\beta_{h} I_{h}}{S_{h}+I_{h}}-\left(d_{h}+\mu_{h}\right) I_{h}
\end{aligned}
$$

- If $\frac{r}{2 \sqrt{\hat{K}}}>d_{h}, \bar{N}_{h}^{*}<\frac{d_{m}}{\alpha c}$, and $S_{h}(0)>S_{h}^{c}$, then the total population of honeybees persists while the healthy mite population $S_{m}$ goes extinct, i.e.,

$$
\limsup _{t \rightarrow \infty} S_{m}(t)=0
$$

where the system (7) is attracted to the healthy-mite-free invariant set $H M F=\left\{\left(S_{h}, I_{h}, S_{m}, I_{m}\right) \in\right.$ $\left.\mathbb{R}_{+}^{4}: S_{m}=0\right\}$ and its dynamics is equivalent to the following three-D system (9):

$$
\begin{aligned}
S_{h}^{\prime} & =\frac{r\left(S_{h}+\rho I_{h}\right)^{2}}{\hat{K}+\left(S_{h}+\rho I_{h}\right)^{2}}-d_{h} S_{h}-\frac{\beta_{h} S_{h} I_{h}}{S_{h}+I_{h}}-\frac{\hat{\beta}_{m h} S_{h} I_{m}}{S_{h}+I_{h}}-\tilde{\beta}_{m h} S_{h} I_{m}-\alpha S_{h} I_{m} \\
I_{h}^{\prime} & =S_{h}\left[\frac{\beta_{h} I_{h}}{S_{h}+I_{h}}+\frac{\hat{\beta}_{m h} I_{m}}{S_{h}+I_{h}}+\tilde{\beta}_{m h} I_{m}\right]-\alpha I_{h} I_{m}-\left(d_{h}+\mu_{h}\right) I_{h} \\
I_{m}^{\prime} & =c \alpha I_{m}\left[I_{h}+S_{h}-\frac{d_{m}+\mu_{m}}{c \alpha}\right] .
\end{aligned}
$$

- Assume that $\frac{r}{2 \sqrt{\hat{K}}}>d_{h}+\beta_{h}+\frac{\hat{\beta}_{m h} N^{*}}{\underline{N}_{h}^{*}}+\left(\tilde{\beta}_{m h}+\alpha\right)\left(N^{*}-\underline{N}_{h}^{*}\right)$ and $S_{h}(0)>S_{h}^{c}$. Then the virus $I=c I_{h}+I_{m}$ persists if the inequality $\frac{\min \left\{\beta_{h}, c \hat{\beta}_{m h}+c \tilde{\beta}_{m h}+c \alpha S_{h}^{*}\right\}}{\max \left\{\left(d_{h}+\mu_{h}\right),\left(d_{m}+\mu_{m}\right)\right\}} \geq 1$ holds.

- Assume that $\frac{r}{2 \sqrt{\hat{K}}}>\frac{d_{h}+\mu_{h}+\alpha N^{*}}{\rho}$ and $N_{h}(0)>\underline{N}_{h}^{c}$. Then the virus $I=c I_{h}+I_{m}$ goes extinct if the 

following inequality holds $\frac{\max \left\{\beta_{h}+\frac{\hat{\beta}_{h m} N^{*}}{N_{h}^{*}}, c \hat{\beta}_{m h}+c \tilde{\beta}_{m h}+c \alpha \bar{N}_{h}^{*}\right\}}{\min \left\{\left(d_{h}+\mu_{h}\right),\left(d_{m}+\mu_{m}\right)\right\}}<1$. Under this condition, the system (7) is attracted to the virus-free invariant set $D F=\left\{\left(S_{h}, I_{h}, S_{m}, I_{m}\right) \in \mathbb{R}_{+}^{4}: I_{h}+I_{m}=0\right\}$ and its dynamics is equivalent to the following two-D model (10)

$$
\begin{aligned}
S_{h}^{\prime} & =\frac{r S_{h}^{2}}{K+S_{h}^{2}}-d_{h} S_{h}-\alpha S_{h} S_{m} \\
S_{m}^{\prime} & =c \alpha S_{h} S_{m}-d_{m} S_{m}
\end{aligned}
$$

Notes: The results of the reduced dynamics in Theorem 3.2 can be easily obtained by the theory of asymptotically autonomous systems [10]. The detailed proof of our results are provided in the last section.

According to Theorem 3.2, the condition $N^{*}=c \frac{\frac{r}{d_{h}}+\sqrt{\left(\frac{r}{d_{h}}\right)^{2}-4 \hat{K}}}{2}<\frac{d_{m}}{\alpha}$ can lead to the extinction of the entire mite population. Therefore, we can conclude that large values for the death rate of mites, $d_{m}$, can lead to the extinction of the colony; and large values of the death rate of mites $d_{m}$, low rates of parasitism $\alpha$, and its energy conversion rate $c$, can lead to either the extinction of the entire mite population $N_{m}$ or the extinction of the healthy mite population $S_{m}$. Here we would like to point out that it is possible to have the persistence of virus infected mites while the healthy mite goes extinct (see the resulting dynamics (9) when the healthy mite goes extinct). In addition, the results of Theorem 3.2 also suggest that: 1 . the persistence of the virus requires a large value for the virus transmission rate between adult honeybees, $\beta_{h}$, or the virus transmission rate between brood and the reproductive mites, $\tilde{\beta}_{m h}$; or the small values of the total death rates of honeybees, $d_{h}+\mu_{h}$, and mites, $d_{m}+\mu_{m} ; \mathbf{2}$. the extinction of the virus requires the small values of all virus transmission rates, i.e., the small values of $\beta_{h}, \hat{\beta}_{m h}, \tilde{\beta}_{m h}, \hat{\beta}_{h m}$; or the large values of the total death rates of honeybees and mites.

Theorem 3.2 provides sufficient conditions that the full system (7) reduces to the virus-free subsystem (10), the mite-free subsystem (8), and the healthy-mite-free subsystem (9). In the following three subsections, we explore the global dynamics of these subsystems.

\subsection{Dynamics of the virus-free subsystem: only parasitism by mites}

Theorem 3.2 in previous section suggests that either low virus transmission rates or large values of the total death rates of honeybees and mites can lead to the extinction of the virus infected honeybees and mites, which gives the following virus-free dynamics (10):

$$
\begin{aligned}
S_{h}^{\prime} & =\frac{r S_{h}^{2}}{\hat{K}+S_{h}^{2}}-d_{h} S_{h}-\alpha S_{h} S_{m} \\
S_{m}^{\prime} & =c \alpha S_{h} S_{m}-d_{m} S_{m}
\end{aligned}
$$

The dynamics of the virus-free system (10) (i.e., the dynamics of the parasitism interactions between honeybees and mites) can be summarized by the following theorem:

Theorem 3.3 (Dynamics of the virus-free subsystem). Let $H^{*}=\frac{d_{m}}{\alpha c}, M^{*}=\frac{1}{\alpha}\left[\frac{r H^{*}}{\hat{K}+\left(H^{*}\right)^{2}}-d_{h}\right]$. The virus-free subsystem (10) can have one, three, or four equilibria. The existence and stability conditions for these equilibria are listed in Table 1. The global dynamics of the virus-free subsystem (10) can be summarized as follows:

1. The system (10) converges to extinction $(0,0)$ for almost all initial conditions if $\frac{r}{2 \sqrt{\hat{K}}}<d_{h}$ or $\frac{d_{m}}{\alpha c}<\bar{N}_{h}^{c}$. 
Table 1: The existence and stability of equilibrium for the virus-free subsystem $(10)$, where $\bar{N}_{h}^{c}=\frac{\frac{r}{d_{h}}-\sqrt{\left(\frac{r}{d_{h}}\right)^{2}-4 \hat{K}}}{2}, \bar{N}_{h}^{*}=$ $\frac{\frac{r}{d_{h}}+\sqrt{\left(\frac{r}{d_{h}}\right)^{2}-4 \hat{K}}}{2}$ and $H^{*}=\frac{d_{m}}{\alpha c}, M^{*}=\frac{1}{\alpha}\left[\frac{r H^{*}}{\hat{K}+\left(H^{*}\right)^{2}}-d_{h}\right]$.

2. If $\bar{N}_{h}^{*}<\frac{d_{m}}{\alpha c}$, depending on initial condition, the trajectory of $(10)$ converges to either $(0,0)$ or $\left(\bar{N}_{h}^{*}, 0\right)$.

3. If $\bar{N}_{h}^{c}<\frac{d_{m}}{\alpha c}<\bar{N}_{h}^{*}$ then Model (10) has a unique interior equilibrium $\left(H^{*}, M^{*}\right)$ which is locally asymptotically stable when $\frac{d_{m}}{\alpha c}>\sqrt{\hat{K}}$ and is a source when $\frac{d_{m}}{\alpha c}<\sqrt{\hat{K}}$.

Notes: Theorem 3.3 provides us a global picture on the dynamics of the virus-free subsystem (10), i.e., the colony virus infected with only mites but not the virus. By applying the results in $[67,71]$, we can conclude that the virus-free subsystem (10) undergoes a subcritical Hopf-bifurcation at $\frac{d_{m}}{\alpha c}=\sqrt{\hat{K}}$. The subsystem (10) has a unique unstable limit cycle around $\left(H^{*}, M^{*}\right)$ whenever $\frac{d_{m}}{\alpha c}<\sqrt{\hat{K}}$. In this case, the periodic orbits expand until it touches the stable manifold of the boundary equilibrium $\left(\bar{N}_{h}^{c}, 0\right)$ which leads to the extinction of both honeybees and the parasitic mites. We refer to this phenomena as a catastrophic event which is similar to CCD. Our theoretical results also suggest that a small death rate for mites and a large parasitism rate can destabilize the system.

Linking to CCD: To illustrate the catastrophic event, we use reasonable parameters from [66, 52]. Let queen's daily egg laying rate in summer be $r=1500$; and the population size of the colony at which queen's daily egg laying rate is half of $r$ be $\sqrt{\hat{K}}=2000$; the natural death rate of honeybees is $d_{h}=0.01$; the parasitism rate is $\alpha=0.005$; the energy conversion rate is $c=0.01$; and the natural death rate of mites is $d_{m}=0.1$. This set of parameter values gives $\frac{d_{m}}{\alpha c}<\sqrt{\hat{K}}$ which implies that a catastrophic event will occur (see Figure 1; the population of honeybees is in red and collapses around time $=200$ day).

Stochastic effects and oscillations: Theorem 3.3 implies that if the inequality $\frac{d_{m}}{\alpha c}<\sqrt{\hat{K}}$ holds, then the virus-free subsystem (10) has a unique unstable limit cycle around $\left(H^{*}, M^{*}\right)$ where for all initial conditions either the system converges to $(0,0)$ quickly or the system experiences the expanding oscillations leading to eventual extinction. The oscillating extinction in the later type is driven by the deterministic dynamics. The extinction fate of the system cannot be prevented by introducing stochastic effects, however, introduced stochastic effects may cause extinction more quickly without expanding oscillations.

Note that $\hat{K}=\frac{K}{\xi_{h}^{2}}$ and $\alpha=\hat{\alpha}\left(1-\xi_{h}\right)\left(1-\xi_{m}\right)$ where $\xi_{h}, \xi_{m}$ are ratio of adult bees and ratio of phoretic mites in the colony, respectively. The catastrophic event occurs when

$$
\frac{d_{m}}{\alpha c}<\sqrt{\hat{K}} \Leftrightarrow \frac{d_{m}}{\hat{\alpha}\left(1-\xi_{m}\right) c \sqrt{K}}<\frac{\left(1-\xi_{h}\right)}{\xi_{h}} \Leftrightarrow \frac{\xi_{h}}{\left(1-\xi_{h}\right)}<\frac{\hat{\alpha}\left(1-\xi_{m}\right) c \sqrt{K}}{d_{m}} .
$$

This inequality provides a critical low hive to brood ratio that can destabilize the system and cause colony sudden extinction. 
Figure 1: Time series (in days) of Model (10) when $r=1500, \alpha=0.005, c=0.01, d_{h}=0.01, d_{m}=0.1$ : population of honeybees is in red while Varroa mites is in blue.

\subsection{Dynamics of the mite-free subsystem: only virus infections}

According to Theorem 3.2, if the honeybee population is too small, e.g., $N^{*}<\frac{d_{m}}{\alpha c}$, then the dynamics of (7) is equivalent to the following mite-free dynamics (8)

$$
\begin{aligned}
S_{h}^{\prime} & =\frac{r\left(S_{h}+\rho I_{h}\right)^{2}}{K+\left(S_{h}+\rho I_{h}\right)^{2}}-d_{h} S_{h}-S_{h} \frac{\beta_{h} I_{h}}{S_{h}+I_{h}} \\
I_{h}^{\prime} & =S_{h} \frac{\beta_{h} I_{h}}{S_{h}+I_{h}}-\left(d_{h}+\mu_{h}\right) I_{h}
\end{aligned}
$$

To continue studying the dynamics of the mite-free system (8), we define $a=\frac{1}{\frac{\beta_{h}}{d_{h}+\mu_{h}}-1}$ as the ratio of the susceptible honeybee population to the virus infected honeybee population; $\mathcal{R}_{0}^{V}=\frac{\beta_{h}}{d_{h}+\mu_{h}}$ as the basic reproduction number, i.e., the number of secondary cases which one case would produce in a completely susceptible population; $\tilde{d}=(a+1) d_{h}+\mu_{h}=d_{h}\left(\frac{\mathcal{R}_{0}^{V}}{\mathcal{R}_{0}^{V}-1}\right)+\mu_{h}$ as the updated average death of the honeybee due to virus infections. In addition, we let $S_{h}^{k}=a I_{h}^{k}, k=1,2$ and

$$
I_{h}^{1}=\frac{\frac{r}{d}-\sqrt{\left(\frac{r}{d}\right)^{2}-4 \frac{\hat{K}}{(a+\rho)^{2}}}}{2}, \quad I_{h}^{2}=\frac{\frac{r}{d}+\sqrt{\left(\frac{r}{d}\right)^{2}-4 \frac{\hat{K}}{(a+\rho)^{2}}}}{2} .
$$

The dynamics of the mite-free system (8) can be summarized by the following theorem:

Theorem 3.4 (Dynamics of the mite-free subsystem). The mite-free subsystem (8) can have one, three, or five equilibria. The existence and stability conditions for these equilibria are listed in Table 2. In addition, the global dynamics of the mite-free subsystem (8) can be summarized as follows:

1. The trajectory of (8) converges to extinction $(0,0)$ for all initial conditions in $\mathbb{R}_{+}^{2}$ if one of the following conditions hold:

$$
\text { - } \frac{r}{2 \sqrt{\hat{K}}}<d_{h} \text {, or }
$$


Table 2: The existence and stability of equilibrium for the mite-free subsystem (8). We have $\mathcal{R}_{0}^{V}=\frac{\beta_{h}}{d_{h}+\mu_{h}}, a=\frac{1}{\mathcal{R}_{0}^{V}-1}, \tilde{d}=$ $(a+1) d_{h}+\mu_{h}=d_{h}\left(\frac{\mathcal{R}_{0}^{V}}{\mathcal{R}_{0}^{V}-1}\right)+\mu_{h}, \bar{N}_{h}^{c}=\frac{\frac{r}{d_{h}}-\sqrt{\left(\frac{r}{d_{h}}\right)^{2}-4 \hat{K}}}{2}, \bar{N}_{h}^{*}=\frac{\frac{r}{d_{h}}+\sqrt{\left(\frac{r}{d_{h}}\right)^{2}-4 \hat{K}}}{2}$, and $I_{h}^{1}=\frac{\frac{r}{d}-\sqrt{\left(\frac{r}{d}\right)^{2}-4 \frac{\hat{K}}{(a+\rho)^{2}}}}{2}, I_{h}^{2}=$ $\frac{\frac{r}{d}+\sqrt{\left(\frac{r}{d}\right)^{2}-4 \frac{\hat{K}}{(a+\rho)^{2}}}}{2}, S_{h}^{k}=a I_{h}^{k}, k=1,2$.

- $\mathcal{R}_{0}^{V}>1$ and $d_{h}<\frac{r}{2 \sqrt{\hat{K}}}<\frac{\tilde{d}}{a+\rho}$.

2. The trajectory of (8) converges to either $(0,0)$ or $\left(\bar{N}_{h}^{*}, 0\right)$ for almost all initial conditions in $\mathbb{R}_{+}^{2}$ if the inequalities $\mathcal{R}_{0}^{V}<1$ and $\frac{r}{2 \sqrt{\hat{K}}}>d_{h}$.

3. The trajectory of (8) converges to either $(0,0)$ or $\left(S_{h}^{2}, I_{h}^{2}\right)$ for almost all initial conditions in $\mathbb{R}_{+}^{2}$ if the inequalities $\mathcal{R}_{0}^{V}>1, \frac{r}{2 \sqrt{\hat{K}}}>\max \left\{d_{h}, \frac{\tilde{d}}{a+\rho}\right\}$ hold.

Notes: Theorem 3.4 implies that the mite-free subsystem (8) has relatively simple dynamics, i.e., no limit cycle. The results show the following interesting findings:

1. Honeybees can persist with proper initial conditions if the virus transmission rate among honeybees $\beta_{h}$ is not large, i.e., $\mathcal{R}_{0}^{V}<1$.

2. Both honeybees and the virus can coexist if $\beta_{h}$ is in the medium range, i.e. $\mathcal{R}_{0}^{V}>1$ and $\frac{\tilde{d}}{a+\rho}<\frac{r}{2 \sqrt{\hat{K}}}$

3. However, the large virus transmission rate among honeybees $\beta_{h}$ can cause colony extinction. This occurs when the inequalities $\mathcal{R}_{0}^{V}>1$ and $d_{h}<\frac{r}{2 \sqrt{\hat{K}}}<\frac{\tilde{d}}{a+\rho}$ hold.

\subsection{Dynamics of the healthy-mite-free subsystem}

According to Theorem 3.2, if $\frac{r}{2 \sqrt{\hat{K}}}>d_{h}, \bar{N}_{h}^{*}<\frac{d_{m}}{\alpha c}$, and $S_{h}(0)>S_{h}^{c}$, then the total population of honeybees persists while the healthy mite population $S_{m}$ goes extinct, i.e.,

$$
\limsup _{t \rightarrow \infty} S_{m}(t)=0
$$

where the system (7) is attracted to the healthy-mite-free invariant set $H M F=\left\{\left(S_{h}, I_{h}, S_{m}, I_{m}\right) \in \mathbb{R}_{+}^{4}\right.$ : $\left.S_{m}=0\right\}$ and its dynamics is equivalent to the following three-D system (9):

$$
\begin{aligned}
S_{h}^{\prime} & =\frac{r\left(S_{h}+\rho I_{h}\right)^{2}}{\hat{K}+\left(S_{h}+\rho I_{h}\right)^{2}}-d_{h} S_{h}-\frac{\beta_{h} S_{h} I_{h}}{S_{h}+I_{h}}-\frac{\hat{\beta}_{m h} S_{h} I_{m}}{S_{h}+I_{h}}-\tilde{\beta}_{m h} S_{h} I_{m}-\alpha S_{h} I_{m} \\
I_{h}^{\prime} & =S_{h}\left[\frac{\beta_{h} I_{h}}{S_{h}+I_{h}}+\frac{\hat{\beta}_{m h} I_{m}}{S_{h}+I_{h}}+\tilde{\beta}_{m h} I_{m}\right]-\alpha I_{h} I_{m}-\left(d_{h}+\mu_{h}\right) I_{h} \\
I_{m}^{\prime} & =c \alpha I_{m}\left[I_{h}+S_{h}-\frac{d_{m}+\mu_{m}}{c \alpha}\right]
\end{aligned}
$$


Let

$$
\begin{aligned}
& f_{1}\left(I_{h}\right)=\frac{\frac{r\left(\frac{d_{m}+\mu_{m}}{c \alpha}-I_{h}+\rho I_{h}\right)^{2}}{\hat{K}+\left(\frac{d_{m}+\mu_{m}}{c \alpha}-I_{h}+\rho I_{h}\right)^{2}}-d_{h} \frac{d_{m}+\mu_{m}}{c \alpha}-\mu_{h} I_{h}}{\alpha\left(\frac{d_{m}+\mu_{m}}{c \alpha}\right)} \\
& f_{2}\left(I_{h}\right)=\frac{\left(\frac{d_{m}+\mu_{m}}{c \alpha}-I_{h}\right)\left[\frac{\beta_{h}}{d_{m}+\mu_{m}}-\left(d_{h}+\mu_{h}\right)\right] I_{h}}{\left.\alpha+\frac{c \alpha \hat{\beta}_{m h}}{d_{m}+\mu_{m}}+\tilde{\beta}_{m h}\right] I_{h}-\frac{d_{m}+\mu_{m}}{c \alpha}\left(\frac{c \hat{\beta}_{m h}}{d_{m}+\mu_{m}}+\tilde{\beta}_{m h}\right)} .
\end{aligned}
$$

The dynamics of the healthy-mite-free subsystem (9) can be summarized by the following theorem:

Theorem 3.5 (Dynamics of the healthy-mite-free subsystem). If $\frac{r}{2 \sqrt{\hat{K}}}>d_{h}$ and $\bar{N}_{h}^{*}<\frac{d_{m}+\mu_{m}}{c \alpha}$, then the population of virus infected mites goes extinct in the subsystem (9), i.e.,

$$
\limsup _{t \rightarrow \infty} I_{m}(t)=0
$$

which reduces to the following mite-free model (8). In addition, the following statements hold

1. If $\left(S_{h}, I_{h}, I_{m}\right)$ is an interior equilibrium of the healthy-mite-free subsystem (9), then $I_{h}$ is a positive intercept of $f_{1}\left(I_{h}\right)$ and $f_{2}\left(I_{h}\right)$ subject to $0<I_{h}<\frac{d_{m}+\mu_{m}}{c \alpha}, S_{h}=\frac{d_{m}+\mu_{m}}{c \alpha}-I_{h}$ and $I_{m}=f_{1}\left(I_{h}\right)$.

2. The healthy-mite-free subsystem (9) has no interior equilibrium if the inequality $r c \alpha<d_{h}\left(d_{m}+\mu_{m}\right)$ holds.

3. Assume that $\frac{r}{2 \sqrt{\hat{K}}}>\frac{d_{h}+\mu_{h}+\alpha N^{*}}{\rho}$ and $N_{h}(0)>\underline{N}_{h}^{c}$. Then both virus infected honeybee population $I_{h}$ and virus infected mites $I_{m}$ persist if the inequalities $\bar{N}_{h}^{*}>\frac{d_{m}+\mu_{m}}{c \alpha}$ and $\mathcal{R}_{0}^{V}=\frac{\beta_{h}}{d_{h}+\mu_{h}}<1$ hold.

Notes: Theorem 3.5 implies that, under the condition of $\bar{N}_{h}^{*}<\frac{d_{m}+\mu_{m}}{c \alpha}$, the virus infected mite population $I_{m}$ goes extinct in the healthy-mite-free subsystem (9) which reduces to the mite-free subsystem (8) that we studied in the previous subsection. In addition, Theorem 3.5 shows that the subsystem (9) has no interior equilibrium if the inequality $r c \alpha<d_{h}\left(d_{m}+\mu_{m}\right)$ holds. Therefore, we could expect the extinction of $I_{m}$ for small values of $r, c, \alpha$ and large values of $d_{h}, d_{m}, \mu_{m}$. This has been confirmed by numerical simulations. The population of honeybees and virus infected mites (9) experiences sudden collapse when we (1) increase the values of $c, \alpha, \hat{K}$, and the related virus transmission rates, or (2) decrease the values of $d_{h}, \rho, r, \mu_{m}$. The biological implications are that increasing or decreasing the values of these parameters destabilizes the system and generates fluctuating dynamics. The destabilizing effects generate unstable oscillations. The amplitudes of oscillations increase until they touch the stable manifold of the extinction equilibrium, which cause the collapse of the colony. The destabilizing effects of $c, \alpha, \hat{K}, d_{m}$ can be explained through the dynamics of the virus free subsystem (10) that we have studied in Theorem 3.3. In addition, our simulations provide important insights on the complicated dynamic of (9), which suggest the following:

- Decreasing the values of $c, \alpha, \hat{K}$ can stabilize the system; small values of $c, \alpha$ can cause the extinction of the virus infected mite population $I_{m}$, and lead to the coexistence of $S_{h}$ and $I_{h}$.

- Increasing the value of $\mu_{h}$ can stabilize the system but large values of $\mu_{h}$ can cause extinction of the colony due to the initial oscillations.

- Decreasing $\mu_{m}$ can destabilize the system; while increasing it can stabilize the system; large values of $\mu_{m}$ can lead to the extinction of $I_{m}$ and the persistence of $S_{h}, I_{h}$.

- Decreasing the value of $\rho$ could destabilize the system, thus causing the extinction of the colony. 
- Increasing the virus transmission rates (i.e., $\beta_{h}, \hat{\beta}_{h m}, \tilde{\beta}_{m h}, \hat{\beta}_{h m}$ ) can stabilize the system, while decreasing their values can destablize the system and cause the extinction of all species.

Synergistic effects of parasitic mites and virus infections: If there are no mites in the system, according to Theorem 3.4, the mite-free subsystem (8) reduces to the healthy honeybee population, i.e., virus goes extinct whenever the initial population of honeybees is above $\bar{N}_{h}^{c}, \frac{r}{2 \sqrt{\hat{K}}}>\frac{d_{h}+\mu_{h}+\alpha N^{*}}{\rho}$, and the basic reproduction number $\mathcal{R}_{0}^{V}=\frac{\beta_{h}}{d_{h}+\mu_{h}}<1$ (see two figures in the first row of Figure 2 where virus infected honeybees go extinct (the black curve in right) and the healthy honeybees persist (the red curve in red)). However, when there are virus infected mites in the colony, both virus and mites can persist under proper conditions (see Figure 3 where virus infected honeybees (the black curve), the healthy honeybees (the red curve), and the virus infected mites persist (the cyan curve) with the healthy mites going extinct (the blue curve). For example, the synergistic effects of parasitic mites and virus infections have been illustrated in Figure 2-3 when $r=1500 ; K=1000000 ; \rho=0.9 ; d_{h}=.15 ; \mu_{h}=0.1 ; \alpha=0.005 ; d_{m}=0.1 ; \mu_{m}=0.01 ; c=$ $0.005 ; \beta_{h}=.24 ; \hat{\beta}_{m h}=0.03 ; \tilde{\beta}_{m h}=.005 ; \hat{\beta}_{h m}=0.03$. These are reasonable parameter values derived from $[66,52]$.
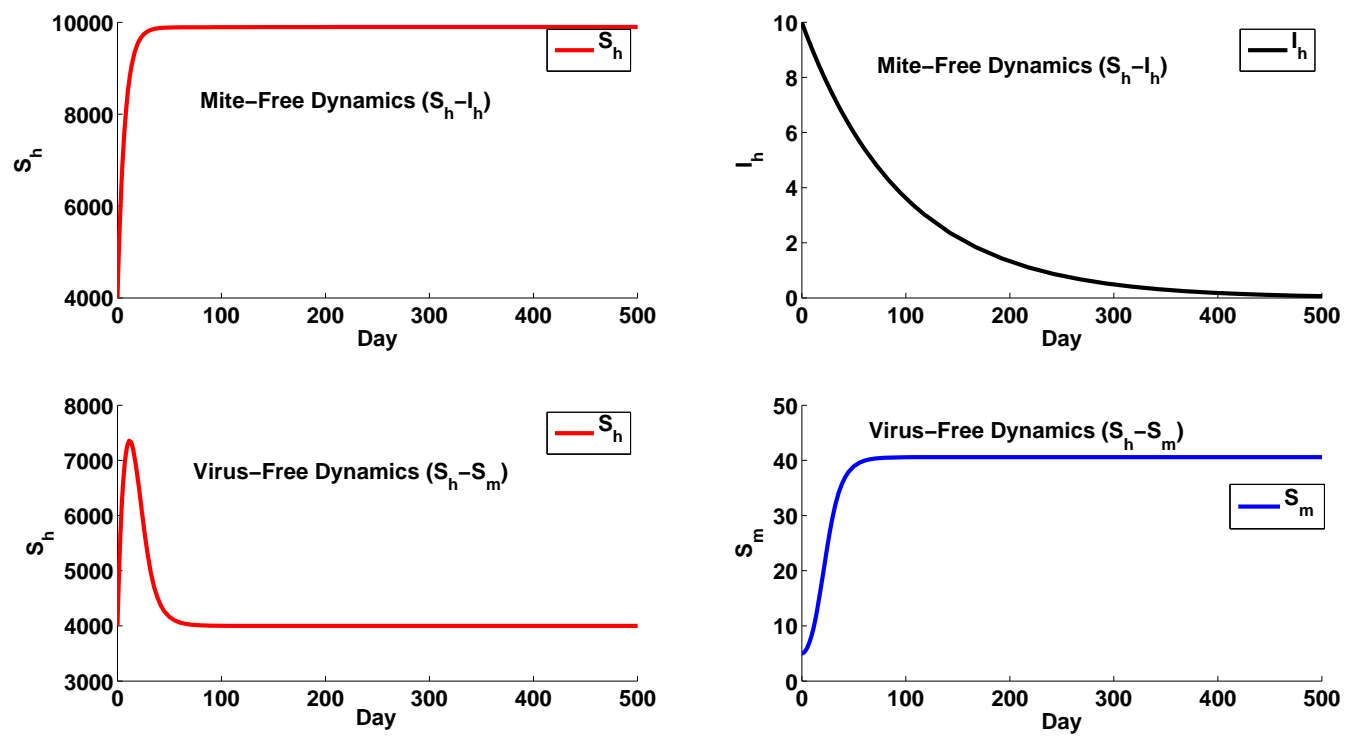

Figure 2: Population dynamics of the subsystems of the the honeybee-mite-virus model (7) when $r=1500 ; K=1000000 ; \rho=$ $0.9 ; d_{h}=.15 ; \mu_{h}=0.1 ; \alpha=0.005 ; d_{m}=0.1 ; \mu_{m}=0.01 ; c=0.005 ; \beta_{h}=.24 ; \hat{\beta}_{m h}=0.03 ; \tilde{\beta}_{m h}=.005 ; \hat{\beta}_{h m}=0.03$. The left figure in the first row is the healthy honeybee population (the red curve) and the right figure in the first row is the virus infected honeybee population (the black curve) in the mite-free subsystem (10) when $S_{h}(0)=4001, I_{h}(0)=10$. The left figure in the second row is the healthy honeybee population (the red curve) and the right figure in the second row is the healthy mite population (the blue curve) in the virus-free subsystem (8) when $S_{h}(0)=4001, S_{m}(0)=5$. 
Figure 3: Population dynamics of the honeybee-mite-virus model $(7)$ when $r=1500 ; K=1000000 ; \rho=0.9 ; d_{h}=.15 ; \mu_{h}=$ $0.1 ; \alpha=0.005 ; d_{m}=0.1 ; \mu_{m}=0.01 ; c=0.005 ; \beta_{h}=.24 ; \hat{\beta}_{m h}=0.03 ; \tilde{\beta}_{m h}=.005 ; \hat{\beta}_{h m}=0.03$ and $S_{h}(0)=4001, I_{h}(0)=$ $10, S_{m}(0)=5, I_{m}(0)=10$. The healthy honeybee population $S_{h}$ is in red; the virus infected honeybee population $I_{h}$ is in black; the healthy mite population $S_{m}$ is in blue; and the virus infected mite population $I_{m}$ is in cyan.

\subsection{Dynamics of the full system}

Recall that the full system (7) of honeybee-mite-virus interactions can be described by the following set of equations:

$$
\begin{aligned}
S_{h}^{\prime} & =\frac{r\left(S_{h}+\rho I_{h}\right)^{2}}{\hat{K}+\left(S_{h}+\rho I_{h}\right)^{2}}-d_{h} S_{h}-\frac{\beta_{h} S_{h} I_{h}}{S_{h}+I_{h}}-\frac{\hat{\beta}_{m h} S_{h} I_{m}}{S_{h}+I_{h}}-\tilde{\beta}_{m h} S_{h} I_{m}-\alpha S_{h}\left(S_{m}+I_{m}\right) \\
I_{h}^{\prime} & =S_{h}\left[\frac{\beta_{h} I_{h}}{S_{h}+I_{h}}+\frac{\hat{\beta}_{m h} I_{m}}{S_{h}+I_{h}}+\tilde{\beta}_{m h} I_{m}\right]-\alpha I_{h}\left(S_{m}+I_{m}\right)-\left(d_{h}+\mu_{h}\right) I_{h} \\
S_{m}^{\prime} & =S_{m}\left[c \alpha S_{h}-\frac{\hat{\beta}_{h m} I_{h}}{S_{h}+I_{h}}-d_{m}\right] \\
I_{m}^{\prime} & =c \alpha\left[I_{h}\left(S_{m}+I_{m}\right)+S_{h} I_{m}\right]+\frac{\hat{\beta}_{h m} I_{h} S_{m}}{S_{h}+I_{h}}-\left(d_{m}+\mu_{m}\right) I_{m}
\end{aligned}
$$

The results from the previous section provide us a complete picture of the dynamics of the subsystems of the full system (7). In this subsection, we explore the dynamics of the full system as the following theorem.

Theorem 3.6 (Colony survival). Assume that $\frac{r}{2 \sqrt{\hat{K}}}>d_{h}$. If $N^{*}<\frac{d_{m}}{\alpha}$ and $\mathcal{R}_{0}^{V}=\frac{\beta_{h}}{d_{h}+\mu_{h}}<1$, the full system (7) converges to the virus-mite-free set DMF $=\left\{\left(S_{h}, I_{h}, S_{m}, I_{m}\right) \in \mathbb{R}_{+}^{4}: S_{m}+I_{h}+I_{m}=0\right\}$ where the system (7) is reduced to the following one-D system (11):

$$
S_{h}^{\prime}=\frac{r S_{h}^{2}}{\hat{K}+S_{h}^{2}}-d_{h} S_{h}
$$

whose dynamics can be summarized as follows:

1. If the inequality $\frac{r}{2 \sqrt{\hat{K}}}<d_{h}$ holds, then (11) converges to 0 . 

falls below $\bar{N}_{h}^{c}$, i.e., $S_{h}(0)<\bar{N}_{h}^{c}$, then (11) also converges to 0 .

Moreover, the following statements hold

1. If the inequalities $\frac{r}{2 \sqrt{\hat{K}}}>\max \left\{d_{h}+\beta_{h}+\frac{\hat{\beta}_{m h} N^{*}}{\underline{N}_{h}^{*}}+\left(\tilde{\beta}_{m h}+\alpha\right)\left(N^{*}-c \underline{N}_{h}^{*}\right), \frac{d_{h}+\mu_{h}+\alpha N^{*}}{\rho}\right\}$ with $N_{h}(0) \geq$ $S_{h}(0)>S_{h}^{c}$ hold, then $S_{h}$ is persistent, i.e., $\liminf _{t \rightarrow \infty} S_{h}(t) \geq S_{h}^{*}$.

2. Assume that $\frac{r}{2 \sqrt{\hat{K}}}>d_{h}+\beta_{h}+\frac{\hat{\beta}_{m h} N^{*}}{\underline{N}_{h}^{*}}+\left(\tilde{\beta}_{m h}+\alpha\right)\left(N^{*}-\underline{N}_{h}^{*}\right)$ with $S_{h}(0)>S_{h}^{c}$ and $\bar{N}_{h}^{*}<\frac{d_{m}}{\alpha c}$ hold. Then the virus $I=c I_{h}+I_{m}$ persists if the inequality $\frac{\min \left\{\beta_{h}, c \hat{\beta}_{m h}+c \tilde{\beta}_{m h}+c \alpha \bar{N}_{h}^{*}\right\}}{\max \left\{\left(d_{h}+\mu_{h}\right),\left(d_{m}+\mu_{m}\right)\right\}} \geq 1$ holds.

3. The full system (7) has no interior equilibrium if one of the following inequalities hold:

$$
\begin{aligned}
& \frac{\beta_{h}}{d_{h}+\mu_{h}}>\frac{\hat{\beta}_{h m}}{d_{m}+\hat{\beta}_{h m}}, \frac{\hat{\beta}_{m h}}{\frac{\alpha \mu_{m} \hat{\beta}_{h m}}{\left(d_{m}+\hat{\beta}_{h m}\right)}+c \alpha \hat{\beta}_{m h}-\tilde{\beta}_{m h} \hat{\beta}_{h m}}>\frac{d_{m}+\mu_{m}}{c \alpha\left(d_{m}+\hat{\beta}_{h m}+\mu_{m}\right)} . \text { Or } \\
& \frac{\alpha \mu_{m} \hat{\beta}_{h m}}{\hat{\beta}_{h m}+d_{m}}>\tilde{\beta}_{m h} \hat{\beta}_{h m}-c \alpha, 0<\frac{\frac{\beta_{h}}{d_{h}+h_{h}}-\frac{\hat{\beta}_{h m}}{\hat{\beta}_{m}+\hat{\beta}_{h m}}}{\left.\beta_{m}+\hat{\beta}_{h m}\right)\left(d_{h}+\mu_{h}\right)}<d_{m} . \text { Or } \\
& \frac{\beta_{h}}{d_{h}+\mu_{h}}<\frac{\hat{\beta}_{h m}}{d_{m}+\hat{\beta}_{h m}}, 0<\frac{\hat{\beta}_{m h}\left(d_{m}+\hat{\beta}_{h m}\right)}{\frac{\alpha \mu_{m} \hat{\beta}_{h m}}{\left(d_{m}+\hat{\beta}_{h m}\right)}+c \alpha \hat{\beta}_{m h}-\tilde{\beta}_{m h} \hat{\beta}_{h m}}<\frac{d_{m}}{c \alpha} . \text { Or } \\
& \frac{\alpha \mu_{m} \hat{\beta}_{h m}}{\hat{\beta}_{h m}+d_{m}}<\tilde{\beta}_{m h} \hat{\beta}_{h m}-c \alpha, \frac{\frac{\beta_{h}}{d_{h}+\mu_{h}}-\frac{\hat{\beta}_{h m}}{\beta_{h}+d_{m}+\hat{\beta}_{h m}}}{\left.d_{h m}\right)\left(d_{h}+\mu_{h}\right)}>\frac{\left(d_{m}+\mu_{m}\right)\left(d_{m}+\hat{\beta}_{h m}\right)}{d_{m}+\hat{\beta}_{h m}+\mu_{m}} . \\
& \text { 4. Assume that } \frac{r}{2 \sqrt{\hat{K}}}>\frac{d_{h}+\mu_{h}+\alpha N^{*}}{\rho} \text { and } N_{h}(0)>\underline{N_{h}^{c}} \text {. Then the total mite population } N_{m}=S_{m}+I_{m} \\
& \text { persists if the inequalities } \bar{N}_{h}^{*}>\frac{d_{m}+\mu_{m}}{c \alpha} \text { and } \mathcal{R}_{0}^{V}=\frac{\beta_{h}}{d_{h}+\mu_{h}}<1 \text { hold. }
\end{aligned}
$$

Notes: Theorem 3.6 along with Theorem $3.1-3.3$, we can conclude that the extinction of virus occurs when all values of all virus transmission rates, $\beta_{h}, \hat{\beta}_{m h}, \tilde{\beta}_{m h}, \hat{\beta}_{h m}$ are small; with the consequence that the full system (7) converges to either $(0,0,0,0)$ or $\left(\bar{N}_{h}^{*}, 0,0,0\right)$ when $\mathcal{R}_{0}^{M}=\frac{\bar{N}_{h}^{*}}{H^{*}}<1$ while (7) converges to either $(0,0,0,0)$ or $\left(H^{*}, 0, M^{*}, 0\right)$ when $1<\mathcal{R}_{0}^{M}<\frac{\bar{N}_{h}^{*}}{S_{h}^{c}}$. The persistence of virus or mites indicates the colony survival even though the population may be low under the influence of virus or mites. Theorem 3.6 provides a summary on sufficient conditions when honeybees can persist in the full (7) alone, with mites, or with virus. The item 4 of Theorem 3.6 is consistent with the results from Theorem 3.5 regarding the synergistic effects of parasitic mites and virus infections: If there are no mites in the system, according to Theorem 3.4, the mite-free subsystem (8) reduces to the only healthy honeybee population, however, in the presence of mites, both virus and mites in the honeybee-mite-virus system (7) can persist under proper conditions (see Figure 2-3 for more details).

The dynamics of the full system (7) can be extremely complicated. We are unable to obtain an explicit form of the interior equilibrium and the related stability. We perform a series of numerical simulations to explore how different parameters affect the population dynamics. The effects of $r, c, \alpha, d_{h}, d_{m}, \mu_{m}, \mu_{h}, \rho$ and virus transmission rates are similar to our observations for the subsystem (9). More specifically, we have the following observations:

1. Effects of $r$ : Increasing $r$ can stabilize the system, but increasing it too much can drive healthy mites $S_{m}$ to extinction while the population of virus infected mites $I_{m}$ increases. Decreasing the values of $r$ can destablize the system, and cause the extinction of mites. And very small values can cause the colony to perish. 
2. Effects of $c$ : Increasing can destabilize the system and cause extinction of all species. Decreasing its value can stabilize the system but extremely small values can drive the extinction of mites.

3. Effects of $\alpha$ : Increasing can destabilize the system and cause extinction of all species while decreasing can stabilize the system and increase the healthy honeybee population. Small values of $\alpha$ can drive the mite population to extinction, and extremely small values can drive both mites and virus to extinction, and only healthy honeybees are left.

4. Effects of $d_{m}$ : Increasing $d_{m}$ can stabilize the system, and drive $S_{m}$ to extinction first. Increasing it further can lead to the extinction of mites, and the system approaches the limiting mite-free system (8). Decreasing can destabilize the system and drive the extinction of the virus. Extremely small values can cause the colony extinction.

5. Effects of $d_{h}$ : Increasing can stabilize the system. Large values can drive virus extinctions, however, extremely large values can lead to the extinction of the colony. Decreasing can destabilize the system which may cause the extinction of the colony under certain conditions.

6. Effects of $\mu_{m}$ : Increasing can drive virus extinction. Extremely large values can lead to the extinction of the colony. Decreasing can destabilize the system. Small values can drive the healthy mites extinct, and extremely small values may cause the extinction of the colony.

7. Effects of $\mu_{h}$ : Increasing can cause the extinction of virus. Decreasing can stabilize the system, and small values can drive healthy mites to extinction.

8. Effects of virus transmission rates: Increasing can destablize the system and cause the extinction of healthy mites $S_{m}$, while extremely large values may drive all populations extinct. Decreasing can cause the extinction of virus.

\subsection{Mechanisms of collapse dynamics and synergistic effects}

Let $r=1500 ; K=1000000 ; \rho=0.9 ; d_{h}=.15 ; \mu_{h}=0.1 ; \alpha=0.005 ; d_{m}=0.1 ; \mu_{m}=0.01 ; c=0.005 ; \beta_{h}=$ $.24 ; \hat{\beta}_{m h}=0.03 ; \tilde{\beta}_{m h}=.005 ; \hat{\beta}_{h m}=0.03$ (Figure 2-3) and $r=1500 ; \rho=0.9 ; \hat{K}=1600001 ; d_{h}=0.15 ; \mu_{h}=$ $0.1 ; \alpha=0.05 ; c=0.005 ; d_{m}=0.1 ; \mu_{m}=0.01 ; \beta_{h}=0.3 ; \hat{\beta}_{m h}=0.08 ; \tilde{\beta}_{m h}=0.001 ; \hat{\beta}_{h m}=0.03$ (Figure 4-5). These are reasonable parameter values derived from $[66,52]$. We use these two sets of parameters as illustrations to explore the synergistic effects of parasite mites and virus infections as well as potential mechanisms linking to CCD (see Figure 1 and Figure 4-5). These comparisons suggest the following:

1. Synergistic effects of parasitic mites and virus infections: Based on the two sets of parameters, we have the following two typical scenarios:

(a) Under the parameter values of $r=1500 ; K=1000000 ; \rho=0.9 ; d_{h}=.15 ; \mu_{h}=0.1 ; \alpha=$ $0.005 ; d_{m}=0.1 ; \mu_{m}=0.01 ; c=0.005 ; \beta_{h}=.24 ; \hat{\beta}_{m h}=0.03 ; \tilde{\beta}_{m h}=.005 ; \hat{\beta}_{h m}=0.03$ :

If there are no mites, the mite-free system (8) (i.e., the dynamics of the healthy (uninfected) honeybee and the virus infected honeybee) converges to only the healthy honeybee with virus infected honeybees becoming extinct (see the first row of Figure 2).

If there is no virus, the virus-free system (10) (i.e., the parasitism dynamics of the healthy honeybee and the healthy mites) converges to a stable equilibrium where both the healthy honeybee and the healthy mites can persist (see the second row of Figure 2).

However, if honeybees, mites, and virus are all present in the system (i.e., the full system (7)), 
then both virus infected honeybees (black curve) and virus infected mites (cyan curve) can persist (see Figure 3).

This implies that the presence of mites can promote the persistence of virus.

(b) Under the parameter values of $r=1500 ; \rho=0.9 ; \hat{K}=1600001 ; d_{h}=0.15 ; \mu_{h}=0.1 ; \alpha=0.05 ; c=$ $0.005 ; d_{m}=0.1 ; \mu_{m}=0.01 ; \beta_{h}=0.3 ; \hat{\beta}_{m h}=0.08 ; \tilde{\beta}_{m h}=0.001 ; \hat{\beta}_{h m}=0.03$ :

If there are no mites, the mite-free system (8) (i.e., the dynamics of the uninfected honeybee and the virus infected honeybee) converges to a stable equilibrium where both infected and uninfected bees can persist (see the first row of Figure 4).

If there is no virus, the virus-free system (10) (i.e., the parasitism dynamics of uninfected bees and mites) converges to the extinction of both species through catastrophic event (see the second row of Figure 2).

However, if honeybees, mites, and virus are all presented in the system (i.e., the full system (7)), then both honeybees and mites go extinct (see Figure 3).

This implies that the presence of the unstable mite population can lead to the extinction of honeybees.
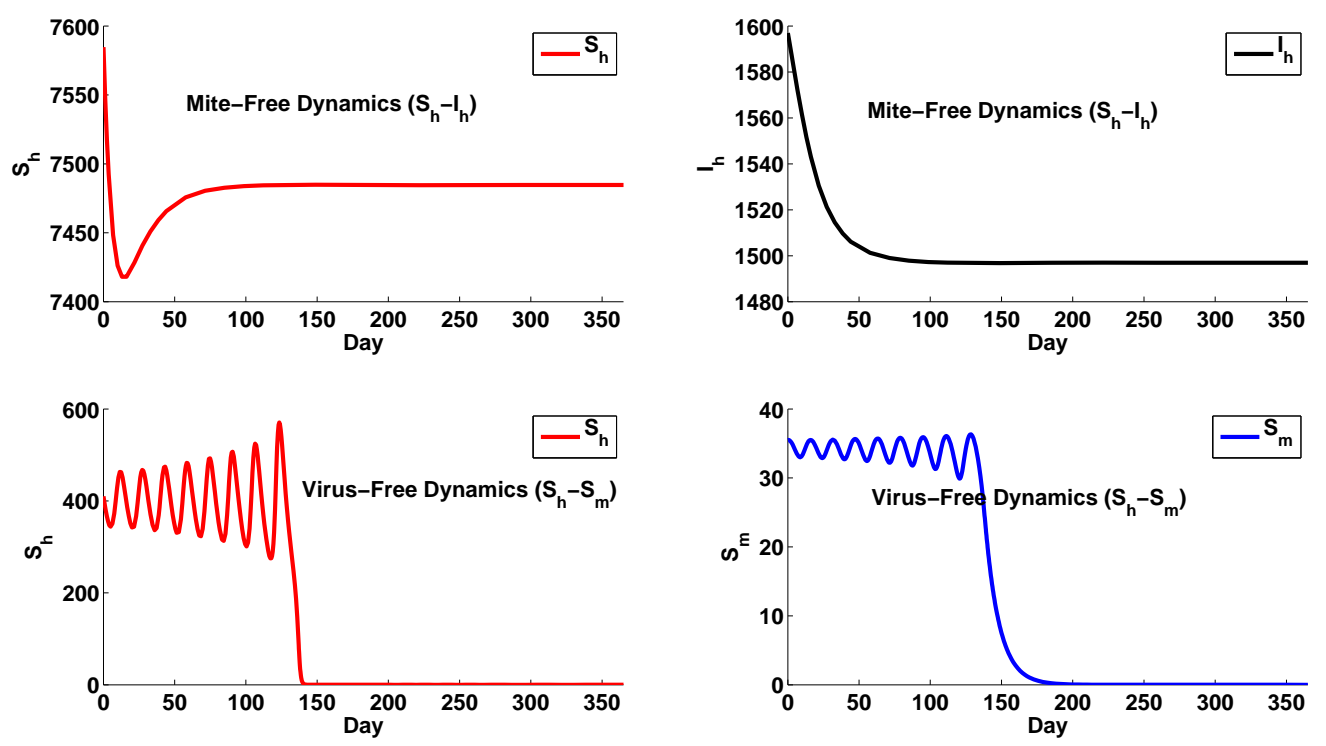

Figure 4: Population dynamics of the subsystems of the the honeybee-mite-virus model (7) when $r=1500 ; \rho=0.9 ; \hat{K}=$ $1600001 ; d_{h}=0.15 ; \mu_{h}=0.1 ; \alpha=0.05 ; c=0.005 ; d_{m}=0.1 ; \mu_{m}=0.01 ; \beta_{h}=0.3 ; \hat{\beta}_{m h}=0.08 ; \tilde{\beta}_{m h}=0.001 ; \hat{\beta}_{h m}=0.03$. The left figure in the first row is the healthy honeybee population (the red curve) and the right figure in the first row is the virus infected honeybee population (the black curve) in the mite-free subsystem (10) when $S_{h}(0)=7684, I_{h}(0)=1700$. The left figure in the second row is the healthy honeybee population (the red curve) and the right figure in the second row is the healthy mite population (the blue curve) in the virus-free subsystem (8) when $S_{h}(0)=410, S_{m}(0)=35$.

2. Linking to CCD: In the absence of virus infections, the subsystem (10) goes through the catastrophic 

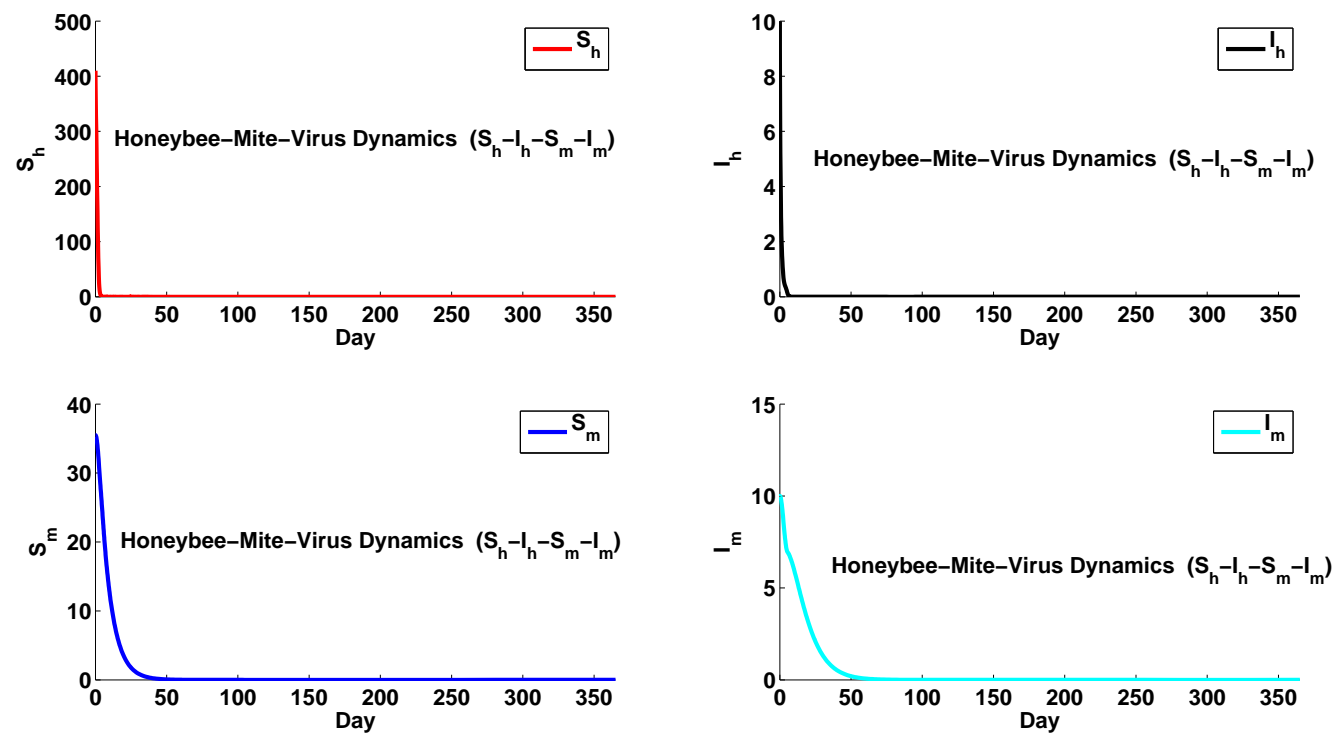

Figure 5: Population dynamics of the honeybee-mite-virus model $(7)$ when $r=1500 ; K=160001 ; \rho=0.9 ; d_{h}=.15 ; \mu_{h}=$ $0.1 ; \alpha=0.05 ; d_{m}=0.1 ; \mu_{m}=0.01 ; c=0.005 ; \beta_{h}=.3 ; \hat{\beta}_{m h}=0.08 ; \tilde{\beta}_{m h}=.001 ; \hat{\beta}_{h m}=0.03$ and $S_{h}(0)=410, I_{h}(0)=$ $10, S_{m}(0)=35, I_{m}(0)=10$. The healthy honeybee population $S_{h}$ is in red; the virus infected honeybee population $I_{h}$ is in black; the healthy mite population $S_{m}$ is in blue; and the virus infected mite population $I_{m}$ is in cyan.

event which causes the extinction of honeybees (see the honeybee population in the red curve of Figure 1). This property has been inherited by the full system (7) as honeybee population goes extinct suddenly for most initial conditions (see the honeybee population in the red curve of the fist left figure in 5).

Our analysis and simulations suggest that it is important to include both mites and virus in studying the population dynamics of honeybees as we proposed the full system (7) due to the synergistic effects of parasitism induced by mites and the virus infections as well as the catastrophic event from the parasitism interactions between mites and honeybees. In other words, if we only consider the honeybee versus virus dynamics as described by the subsystem (8), or only consider the parasitism interactions between mites and honeybees as described by the subsystem (10), we are not able to capture the full mechanics that can lead to colony death or the biological implications on the persistence of virus in colonies. In addition, the full system (7) has rich dynamics which can possess multiple attractors. Thus, depending on initial conditions, the full system (7) can either experience extinction or have coexistence of both honeybees and mites. More sophisticated mathematical analysis is needed in order to understand the detailed dynamics.

\section{Discussion}

The association of virus infection with Varroa mites infestation in honeybee colonies causes great concern for researchers and beekeepers [12]. Many studies have suggested that Varroa mite infestations could be a key explanatory factor for the widespread increase in annual honeybee colony mortality and has been implicated as a contributing factor leading to CCD [43]. In this paper, we derive and study a honeybeevirus-mite model by using the susceptible-infectious (SI) disease framework. Our proposed model uses frequency-dependent transmission functions to model horizontal virus transmissions between the phoretic mites and honeybees, a Holling Type I functional response to model parasitic interactions between mites 
and honeybees, a density-dependent transmission function to model the vertical virus transmission between mites and honeybees during the mite reproductive phase and a frequency-dependent transmission function to model the horizontal virus transmission among honeybees. Our analytical and numerical results of the full system suggest:

1. Initial honeybee populations play an important role in its persistence since its dynamics exhibits strong Allee effects in the absence of both parasites and virus (see Theorem 3.1). In addition, patterns of population dynamics are sensitive to initial conditions as suggested by our numerical results, i.e., depending on initial conditions, the full system can experience the catastrophic event where honeybees collapse dramatically, or both mites and honeybees can coexist but may exhibit different dynamical patterns.

2. In the absence of Varroa mites, the honeybee and virus system has only equilibrium dynamics (see Theorem 3.4 and the first row of Figure 2-3). In the presence of Varroa mites, the synergistic effects of parasitism and virus make virus and mites more persistent (see Theorem 3.5-Theorem 3.6), and thus, difficult to control (see Theorem 3.2 and the related arguments). In addition, the synergistic effects could stabilize the system, but could also drive both honeybees and mites go extinct (see Figure 5).

3. In the absence of virus infections, the mite and honeybee system can be destabilized by the low adult workers to brood ratio in the colony where the system has oscillating dynamics leading to a sudden extinction of all species (see Theorem 3.3 and Figure 1). This dynamical property is called the catastrophic event which has been inherited by the full system when the virus infection is present (see Figure 5). This phenomenon could be linked to CCD which has been observed in honeybee colonies where the low hive to brood ratio could be a contributing factor.

4. Our numerical simulations suggest that large values of virus transmission rates can drive the extinction of healthy mites while extremely large values can lead to the extinction of all species.

Identify the contribution to the broader field: Our current work provides many useful insights regarding the complicated synergistic effects that Varroa and associated virus infections have on honeybee population dynamics. The model generates scenarios frequently seen in colonies and provides insights into the limitations of certain Varroa control strategies in preventing colony death especially over winter or in early spring. Our theoretical results imply that maintaining the adult workers to brood ratio above a critical threshold during brood rearing periods (e.g, spring and summer) is pivotal for colony survival. These results are similar to previous reports (e.g., [58]). Our work suggests that increasing the values of the queen egg laying rate and the mortality rates of mites can stabilize the system. However, a unique feature to our model is that increase colony population growth also potentially increase Varroa and virus populations. The behavior of the full model generates dynamics commonly seen in the field where large colonies die overwinter even when mite populations are reduced with miticide treatments in the fall [54]. In our model, virus transmission can occur during both contacts with nestmates and parasitism events so viruses do not go extinct in the absence of Varroa as in Ratti et al. [52]. The addition of vertical transmission of virus between nestmates generates dynamics that differ from those in previous models where it can take several years for colonies to die. Those models assume that reductions in Varroa populations result in simultaneous reductions in virus levels. However, this is not realistic since the bees can harbor viruses and transmit them among nestmates. Our model generates dynamics seen in the field where colonies collapse overwinter due to high virus transmission rates and the persistence of virus even in the absence of Varroa [26]. These findings suggest that colony losses are due to the combination of virus transmission between nestmates in addition to parasitism and that late season Varroa treatments might not insure overwintering colony survival. 

of time if they are infected with either viruses or Varroa mites. However, the combination of Varroa mites and viruses inevitably leads to colony collapse as shown in the full model. Parasites and pathogens that ultimately kill their hosts must adapt strategies that enable them to disperse before the host dies. Mites cannot move between colonies on their own, but they can disperse by attaching to foragers leaving the hive. If the forager drifts to another colony, the mite and virus have successfully dispersed. Viruses transmitted by Varroa mites (e.g., Deformed wing virus and Israeli acute paralysis virus) affect learning and memory and might affect the return rate of infected foragers to their colonies [36, 28, 33]. Mite migration into colonies on drifting foragers and from the robbing of collapsing colonies has been documented [56, 24, 23, 22]. The synergistic effects revealed in our honeybee-mite-virus system that ultimately lead to colony extinction provide a mathematical explanation for dispersal strategies that might include mite migration when both viruses and Varroa mites are present.

Compare the findings with other works: As we mentioned in our introduction, the work of Sumpter and Martin [66] and Ratti et al. [52] are most relevant modeling papers for our study proposes. They have done great work with interesting results. However, there are limitations on assumptions of their models. Sumpter and Martin [66] assumed that the mites population is constant and the virus transmission occurs only through Varroa mites. This assumption prevents us to study how mites population affect the virus transmission and the honeybee population dynamics. The model of Ratti et al. [52] assumes that the virus transmission does not transmit through honeybees themselves. This is not realistic. Because this assumption implies that the extinction of Varroa mites can lead to the extinction of virus, which cannot reflect the fact that honeybee colonies have viruses in the absence of mites. Our honeybee-mite-virus model has relaxed these unrealistic assumptions used in the work of Sumpter and Martin [66] and Ratti et al. [52]. More specifically, our proposed model includes the bee to bee virus transmission and both population dynamics of virus and mites with implicit age structures. By including the bee to bee virus transmission, our model allows us to explore the effects of virus and mites on honeybee population dynamics separately. This is one improvement by comparing to the model of Ratti et al. [52]. This allows us to investigate the dynamics of the mites-honeybee interactions (i.e., the virus-free dynamics (10)). Our study of the virus-free dynamics (10) implies that the large values of the parasitism rate of mites and the nutrient conversion rate from honeybee to mite and the small value of the mite natural mortality can destabilize the system to generate a catastrophic event that is similar to CCD. Our model permits us to study the dynamics of the virus-honeybee interactions (i.e., the mite-free dynamics (8)) which has only equilibrium dynamics. The comparison of the virus-free dynamics (10) and the mite-free dynamics (8) suggests that the parasitism from mites should be a key factor contributing CCD, not virus infections.

The second improvement of our model is that our model includes both population dynamics of virus and mites with implicit age structure. This improvement allows us to explore the synergistic effects of Varroa mites and the associated virus infections on the honeybee population dynamics. Our study suggests that (a) the presence of mites is able to promote the persistence of virus; (b) the presence of the unstable mite population can lead to the extinction of honeybees while in the absence of mites, honeybee and virus are able to coexist; (c) the presence of both mites and virus can lead to complicated dynamics which can possess multiple attractors, i.e., depending on initial conditions, the full system can either experience extinction or have coexistence of both honeybees and mites; and (d) the low hive to brood ratio could be a contributing factor of CCD. In addition, our model is able to provide sufficient conditions that can guarantee the persistence of honeybee population and conditions that lead to the extinction of honeybees.

There are models where pathogens are introduced via the foragers and spread through the colony by worker-worker interactions only $[34,8]$. These models do not include sensitivity to Varroa mites. The behaviors of these models are similar to the case in our analysis examining population dynamics of the mite-free system with virus only. The model by Betti et al. [8] simulates the interplay between the dynamics of the spread of the pathogen and the changing demographics of the colony during the season. The model reveals a 
critical period just prior to the onset of winter where if a contagious pathogen is introduced, the colony will collapse because the number of bees surviving the winter is not sufficient to support colony growth in the spring. In our model, the mite free subsystem with only virus infections causes colony collapse when transmission rates are high as they are during winter colony confinement. These models provide mathematical explanations for the loss of colonies from pathogens alone over the winter due to adult bee to brood ratios in the spring that are insufficient to generate colony growth.

Models simulating the effects on colony survival of Varroa alone generate predictions of a slow decline in colony populations followed by a rapid collapse [15]. These predictions are similar to the catastrophic event demonstrated in our model analysis with the virus-free system. The behaviors of the models are similar to documented colony losses from Varroa where strong colonies die rapidly in the spring [54]. The initial conditions of colony size, egg laying rates, and Varroa infestation levels strongly influence the length of time required for colonies to collapse. If initial mite levels are low, it can take more than a year for colonies to collapse. Models have been constructed to combine Varroa and viruses that can be spread throughout a colony by Varroa feeding and reproductive behaviors $[66,52,6]$. In the model by Sumpter and Martin [66], the Varroa population is constant. Other models simulate the growth of the Varroa population over time either as a function of colony growth $[15,6]$ or with a simple logistic equation [52]. Mortality can occur from the effects of parasitism or viral infection. These models reveal the interplay between life stages when death can occur due to infection and colony survival, and capture a counterintuitive aspect of viral spread. Viruses that are highly virulent require greater proportions of Varroa infected with the virus (because transmission rates are reduced), or a longer time to reach epidemic levels that cause colony death $[66,52,8]$. An increase in the transmission rate, simulated by bees being infected at an earlier age, also has a highly deleterious effect on colony survival. Our model predicts colony death when virus transmission rates are high or colony growth is limited by egg laying. In all models cited above, there is persistence in virus infection due to the presence of Varroa.

The results of our model analyses indicate that Varroa control strategies need to keep mite levels and virus transmission rates low, while maintaining adult to brood ratios that insure colony growth. This can be achieved by reducing mite populations in the early spring when brood rearing resumes. Initial conditions strongly influence late season mite population size and virus levels, so mite treatments in early spring can reduce mite population numbers and growth rates. Keeping mite and virus levels low will prevent high levels of parasitism and viral infections later in the year particularly before colonies overwinter. The bees that emerge prior to the end of the yearly brood rearing period should be free of parasitism and pathogen stress. The longevity of bee comprising the overwintering population ultimately determines brood rearing rates and colony growth in the spring. The model suggests that the effectiveness of miticide treatments applied late in the fall when Varroa populations peak might be limited because virus levels and transmission rates will be high in the winter cluster. In the analysis of the full model, high virus transmission rates reduce the adult to brood ratio in the spring when brood rearing resumes leading to colony extinction.

Limitation of the current model: In our current model, we assume that the ratios of brood and adult are constants as a replacement for having the explicit dynamical age structure profile. This simplification allows us to obtain rigorous results on how this ratio affect colony dynamics. This model is a good description of the dynamics of the system at or near the steady-state of the age-structured system, but not far from steady-state. This limitation implies that our current model may not be a good description of how colony dynamics will progress if there is a sudden change in the colony demography, such as might occur during a colony collapse event. In particular, the importance of a dynamics age profile of a colony to whether or not it will collapse has been highlighted by the recent work by Perry et al. [50], in which the age of onset of foraging is an important factor in determining colony survival. The work of Perry et al. [50] examined the social dynamics underlying the dramatic colony failure with an aid of a honeybee population model. Their model does not include population dynamics of Varroa mites or virus infections, but it does includes bee foraging performance varying with age, and displays dynamics of colony population collapse that are similar 
to field reports of CCD. It is important to expand our current modeling framework to include the detailed stage-structure model of honeybees with social dynamics, age of onset of foraging as the work by Perry et al. $[50]$.

Suggest options for further research: A common feature to all the models described above is that equilibrium states between colony populations and pathogens are likely to be unstable in many situations. Without beekeeper intervention, colonies infested with Varroa and infected with viruses die. If colonies with Varroa and viruses are ultimately coursing toward extinction, the pathogens and parasites must adapt dispersal strategies prior to complete colony collapse. The migration of mites among colonies is well documented and appears to be a significant factor in the growth of the Varroa populations particularly in the fall $[56,24,23,22]$. The inclusion of migration of mites with disease into and out of colonies is needed to provide an added dimension of reality to models that simulate the growth of Varroa populations and the spread of disease in colonies. Models that simulate mite population growth from reproduction alone might ultimately overestimate the time required for colonies to collapse and be poor representation of present conditions particularly in commercial apiaries.

A fixed ratio of adult workers to brood proposed in our current model is a simplification since this ratio varies with the availability of nutrients, the quality and availability of pollen. The mortality of honey bees depends on different risk factors such as parasites, pathogen, viruses, pesticides, nutrition and environmental changes [64]. Among these factors, honey bee nutrition is one of the main factors which impacts honey bee health and influences the capabilities of honey bees to combat different stressors. Nutrients such as protein from pollen is essential in fighting parasites and viruses, and in maintaining the high adult worker to brood ratio in honeybees. This is because honeybees solely depend on protein (containing essential amino acids, vitamins and mineral) and energy, respectively [3]. Cage studies showed that honey bees could survive for a long time without pollen provision [5], but pollen feeding significantly prolonged their lifespan $[38,42,61,60]$. Intensive research has investigated the connection between nutrition and honey bee virus and stress resistance. Both cage studies and field studies indicated that bees with poor nutrition were under more stress (Wang et al. unpublished data) [72], more susceptible to Nosema and Varroa destructor, and had shorter lifespan $[19,53]$. DeGrandi-Hoffman found a similar result that bees with good pollen or protein nutrition were more resistant to many different viruses [14]. Furthermore, studies on molecular mechanisms suggested that pollen nutrition may positively affect antimicrobial peptides and improve immune defensive response to parasites [17]. The additions of nutrient/brood dynamics and explicit division of labor would generate scenarios where nutritional stress could reduces immune function resulting in colony death from the proliferation of viruses and other pathogens and thus more closely align with the general consensus that colony losses have multifactorial origins [45]. Therefore, a more realistic model that includes nutrient/brood dynamics with explicit division of labor are needed in addition to migration.

Moreover, seasonal effects are also important. Models that simulate colony growth either assume a constant egg laying rate or have sensitivity to seasonal patterns of brood production $[15,52,55,6,8,51]$. If seasonal effects on egg laying are included, the worker to brood ratio varies throughout the yearly cycle as does the vulnerability of the colony. If parasites such as Varroa or pathogens such as viruses are components in a model, then including seasonal effects on egg laying prevents an overestimation of the mite load and population size of uninfected (i.e., healthy bees) that can be maintained without affecting colony survival. In future work, a realistic model should also include seasonal effects. 


\section{Proof}

\section{Proof of Theorem 3.1}

Proof. According to Theorem A.4 (p.423) of Thieme (2003), we can conclude that Model (7) is positive invariant in $X$. Let $d=\min \left\{d_{h}, d_{m}\right\}, N_{h}=S_{h}+I_{h}, N_{m}=S_{m}+I_{m}$, and $N=c N_{h}+N_{m}$. Then we have

$$
\begin{aligned}
N^{\prime}=c N_{h}^{\prime}+N_{m}^{\prime} & =\frac{r c\left(S_{h}+\rho I_{h}\right)^{2}}{\hat{K}+\left(S_{h}+\rho I_{h}\right)^{2}}-c d_{h} N_{h}-c \mu_{h} I_{h}-d_{m} N_{m}-\mu_{m} I_{m} \\
& \leq \frac{r c(N / c)^{2}}{\tilde{K}+(N / c)^{2}}-\min \left\{d_{h}, d_{m}\right\}\left(c N_{h}+N_{m}\right)=\frac{r c N^{2}}{c^{2} \tilde{K}+N^{2}}-\min \left\{d_{h}, d_{m}\right\} N=\frac{r c N^{2}}{c^{2} \tilde{K}+N^{2}}-d N
\end{aligned}
$$

which implies that $\limsup _{t \rightarrow \infty} N(t) \leq c \frac{\frac{r}{\frac{r}{d}+\sqrt{\left(\frac{r}{d}\right)^{2}-4 \hat{K}}}}{2}=N^{*}$ with implication that $\limsup _{t \rightarrow \infty} N(t)=0$ if either $\frac{r}{d}<2 \sqrt{\hat{K}}$ or $N(0)<N^{c}=c \frac{\frac{r}{d}+\sqrt{\left(\frac{r}{d}\right)^{2}-4 \hat{K}}}{2}$ holds. The arguments above also imply that if $\frac{r}{d}>2 \sqrt{\hat{K}}$, then we have

$$
\limsup _{t \rightarrow \infty} N_{h}(t) \leq N^{*} / c \text { and } \limsup _{t \rightarrow \infty} N_{m}(t) \leq N^{*}
$$

Similarly, we have follows for $N_{h}$ :

$$
\begin{aligned}
N_{h}^{\prime}=S_{h}^{\prime}+I_{h}^{\prime} & =\frac{r\left(S_{h}+\rho I_{h}\right)^{2}}{\hat{K}+\left(S_{h}+\rho I_{h}\right)^{2}}-d_{h} N_{h}-\mu_{h} I_{h}-\alpha N_{h} N_{m} \\
& \leq \frac{r N_{h}^{2}}{\hat{K}+N_{h}^{2}}-d_{h} N_{h}
\end{aligned}
$$

which implies that

$$
\limsup _{t \rightarrow \infty} N_{h}(t) \leq \frac{\frac{r}{d_{h}}+\sqrt{\left(\frac{r}{d_{h}}\right)^{2}-4 \hat{K}}}{2}=\bar{N}_{h}^{*} \text { if } \frac{r}{d_{h}}>2 \sqrt{\hat{K}}
$$

and

$$
\limsup _{t \rightarrow \infty} N_{h}(t)=0 \text { if } \frac{r}{d_{h}}<2 \sqrt{\hat{K}} \text { or } N_{h}(0)<S_{h}^{c}=\frac{\frac{r}{d_{h}}-\sqrt{\left(\frac{r}{d_{h}}\right)^{2}-4 \hat{K}}}{2} .
$$

On the other hand, we have

$$
\begin{aligned}
N_{h}^{\prime}=S_{h}^{\prime}+I_{h}^{\prime} & \geq \frac{r \rho^{2}\left(S_{h} / \rho+I_{h}\right)^{2}}{\hat{K}+\rho^{2}\left(S_{h} / \rho+I_{h}\right)^{2}}-\left(d_{h}+\mu_{h}+\alpha N_{m}\right) N_{h} \\
& \geq \frac{r \rho^{2} N_{h}^{2}}{\hat{K}+\rho^{2} N_{h}^{2}}-\left(d_{h}+\mu_{h}+\alpha N^{*}\right) N_{h} \\
& \geq \frac{r N_{h}^{2}}{\hat{K} / \rho^{2}+N_{h}^{2}}-\left(d_{h}+\mu_{h}+\alpha N^{*}\right) N_{h}
\end{aligned}
$$

Therefore, apply the comparison theorem, we can conclude that

$$
\liminf _{t \rightarrow \infty} N_{h}(t) \geq \frac{\frac{r}{d_{h}+\mu_{h}+\alpha N^{*}}+\sqrt{\left(\frac{r}{d_{h}+\mu_{h}+\alpha N^{*}}\right)^{2}-4 \hat{K} / \rho^{2}}}{2}=\underline{N}_{h}^{*}
$$


if the following inequalities hold

$$
\frac{r}{d_{h}+\mu_{h}+\alpha N^{*}}>2 \sqrt{\frac{\hat{K}}{\rho^{2}}} \text { and } N_{h}(0)>\underline{N}_{h}^{c}=\frac{\frac{r}{d_{h}+\mu_{h}+\alpha N^{*}}-\sqrt{\left(\frac{r}{d_{h}+\mu_{h}+\alpha N^{*}}\right)^{2}-4 \hat{K} / \rho^{2}}}{2} .
$$

The discussions above provide sufficient conditions that allow $N_{h}$ being persistent, i.e., when the inequalities $\frac{r}{d_{h}+\mu_{h}+\alpha N^{*}}>2 \sqrt{\frac{\hat{K}}{\rho^{2}}}$ and $N_{h}(0)>\underline{N}_{h}^{c}$ hold. This implies that $S_{h}$ is also persistent under this condition since all species go extinct if $S_{h}$ goes extinct. However, this persistence condition does not provide an estimate of $S_{h}$. To explore an estimation of $S_{h}$, we look at the population of $S_{h}$.

Recall that if $\frac{r}{d_{h}+\mu_{h}+\alpha N^{*}}>2 \sqrt{\frac{\hat{K}}{\rho^{2}}}$ and $N_{h}(0)>\underline{N}_{h}^{c}$, then we have the following inequalities

$$
\underline{N}_{h}^{*} \leq \liminf _{t \rightarrow \infty} N_{h}(t) \leq \liminf _{t \rightarrow \infty} N(t) / c \leq \limsup _{t \rightarrow \infty} N(t) / c \leq N^{*} / c .
$$

If the inequalities $\frac{r}{d_{h}+\beta_{h}+\frac{\hat{\beta}_{m h} N^{*}}{\underline{N}_{h}^{*}}+\left(\tilde{\beta}_{m h}+\alpha\right)\left(N^{*}-c \underline{N}_{h}^{*}\right)}>2 \sqrt{\hat{K}}$ and

$$
S_{h}(0)>\frac{\frac{r}{d_{h}+\beta_{h}+\frac{\beta_{m h} N^{*}}{N_{h}^{*}}+\left(\tilde{\beta}_{m h}+\alpha\right)\left(N^{*}-c N_{h}^{*}\right)}-\sqrt{\left(\frac{r}{d_{h}+\beta_{h}+\frac{\beta_{m h} N^{*}}{N_{h}^{*}}+\left(\tilde{\beta}_{m h}+\alpha\right)\left(N^{*}-c N_{h}^{*}\right)}\right)^{2}-4 \hat{K}}}{2}
$$

hold, then we have following inequalities

$$
\begin{aligned}
S_{h}^{\prime} & =\frac{r\left(S_{h}+\rho I_{h}\right)^{2}}{\hat{K}+\left(S_{h}+\rho I_{h}\right)^{2}}-d_{h} S_{h}-\frac{\beta_{h} S_{h} I_{h}}{S_{h}+I_{h}}-\frac{\hat{\beta}_{m h} S_{h} I_{m}}{S_{h}+I_{h}}-\tilde{\beta}_{m h} S_{h} I_{m}-\alpha S_{h}\left(S_{m}+I_{m}\right) \\
& >\frac{r\left(S_{h}\right)^{2}}{\hat{K}+\left(S_{h}\right)^{2}}-d_{h} S_{h}-\beta_{h} S_{h}-\frac{\hat{\beta}_{m h} I_{m}}{N_{h}} S_{h}-\tilde{\beta}_{m h} S_{h} N_{m}-\alpha S_{h} N_{m} \\
& >\frac{r\left(S_{h}\right)^{2}}{\hat{K}+\left(S_{h}\right)^{2}}-\left[d_{h}+\beta+\frac{\hat{\beta}_{m h} N^{*}}{\underline{N}_{h}^{*}}+\left(\tilde{\beta}_{m h}+\alpha\right) N_{m}\right] S_{h} \\
& =\frac{r\left(S_{h}\right)^{2}}{\hat{K}+\left(S_{h}\right)^{2}}-\left[d_{h}+\beta+\frac{\hat{\beta}_{m h} N^{*}}{\underline{N}_{h}^{*}}+\left(\tilde{\beta}_{m h}+\alpha\right)\left(N-c N_{h}\right)\right] S_{h} \\
& >\frac{r\left(S_{h}\right)^{2}}{\hat{K}+\left(S_{h}\right)^{2}}-\left[d_{h}+\beta+\frac{\hat{\beta}_{m h} N^{*}}{\underline{N}_{h}^{*}}+\left(\tilde{\beta}_{m h}+\alpha\right)\left(N^{*}-c \underline{N}_{h}^{*}\right)\right] S_{h}
\end{aligned}
$$

which implies that

$$
\liminf _{t \rightarrow \infty} S_{h}(t) \geq S_{h}^{*}=\frac{\frac{r}{d_{h}+\beta_{h}+\frac{\hat{\beta}_{m h} N^{*}}{\underline{N}_{h}^{*}}+\left(\tilde{\beta}_{m h}+\alpha\right)\left(N^{*}-c \underline{N}_{h}^{*}\right)}+\sqrt{\left(\frac{r}{d_{h}+\beta_{h}+\frac{\hat{\beta}_{m h} N^{*}}{\underline{N}_{h}^{*}}+\left(\tilde{\beta}_{m h}+\alpha\right)\left(N^{*}-c \underline{N}_{h}^{*}\right)}\right)^{2}-4 \hat{K}}}{2} .
$$

Therefore, we can conclude that $S_{h}$ is persistent with the following properties:

$$
S_{h}^{*} \leq \liminf _{t \rightarrow \infty} S_{h}(t) \leq \liminf _{t \rightarrow \infty} N_{h}(t) \leq \liminf _{t \rightarrow \infty} \frac{N(t)}{c} \leq \limsup _{t \rightarrow \infty} N(t) / c \leq N^{*} / c
$$

if the following inequalities hold

1. $\frac{r}{2 \sqrt{\hat{K}}}>\max \left\{d_{h}+\beta_{h}+\frac{\hat{\beta}_{m h} N^{*}}{\underline{N}_{h}^{*}}+\left(\tilde{\beta}_{m h}+\alpha\right)\left(N^{*}-\underline{N}_{h}^{*}\right), \frac{d_{h}+\mu_{h}+\alpha N^{*}}{\rho}\right\}$ with $N_{h}(0) \geq S_{h}(0)>S_{h}^{c}$. 
It is easy to check that the extinction equilibrium $E_{0}=(0,0,0,0)$ is always local stable. We omit the details. Based on the discussion of the upper bound of the total population $N$ and the total population of honeybees $N_{h}$, we can conclude that the system (7) converges to $E_{0}$ globally if $2 \sqrt{\hat{K}}>\frac{r}{d_{h}}$ holds; while if the initial population satisfies either $N(0)<N^{c}$ or $N_{h}(0)<\bar{N}_{h}^{c}$, then the system (7) converges to $E_{0}$ locally.

\section{Proof of Theorem 3.2}

Proof. Now we consider the population of $N_{m}$. From Model (7), we obtain the following inequalities:

$$
c \alpha N_{h} N_{m}-\left(d_{m}+\mu_{m}\right) N_{m} \leq N_{m}^{\prime}=S_{m}^{\prime}+I_{m}^{\prime}=c \alpha N_{h} N_{m}-d_{m} N_{m}-\mu_{m} I_{m} \leq N_{m}\left(c \alpha N_{h}-d_{m}\right)
$$

which implies that if $N^{*}<\frac{d_{m}}{\alpha}$, then we have

$$
\begin{aligned}
N_{m}^{\prime} & \leq N_{m}\left[c \alpha N_{h}-d_{m}\right]=N_{m}\left[c \alpha\left(N-N_{m}\right) / c-d_{m}\right] \\
& \leq N_{m}\left[\alpha N^{*}-d_{m}-\alpha N_{m}\right]<N_{m}\left[\alpha N^{*}-d_{m}\right]
\end{aligned}
$$

This indicates that $\lim \sup _{t \rightarrow \infty} N_{m}(t)=0$.

Assume that the following inequalities hold $\frac{r}{d_{h}}>2 \sqrt{\hat{K}}$, and $S_{h}(0)>S_{h}^{c}$. Then according to Theorem 3.1 , we have

$$
\limsup _{t \rightarrow \infty} S_{h}(t) \leq \limsup _{t \rightarrow \infty} N_{h}(t) \leq \bar{N}_{h}^{*}
$$

Now let us focus on the population of $S_{m}$. Notice that we have the following inequalities when time is large enough,

$$
S_{m}^{\prime}=S_{m}\left[c \alpha S_{h}-\frac{\hat{\beta}_{h m} I_{h}}{S_{h}+I_{h}}-d_{m}\right] \leq S_{m}\left[c \alpha N_{h}-d_{m}\right] \leq S_{m}\left[c \alpha \bar{N}_{h}^{*}-d_{m}\right] .
$$

This implies that if $\bar{N}_{h}^{*}<\frac{d_{m}}{c \alpha}$ and $\frac{r}{d_{h}}>2 \sqrt{\hat{K}}$, and $S_{h}(0)>S_{h}^{c}$, the healthy mite population $S_{m}$ goes extinct while the total population of honeybees persists, i.e.,

$$
\limsup _{t \rightarrow \infty} S_{m}(t)=0 .
$$

Now we look at the population dynamics of $I_{m}$ and $I_{h}$. Let $I=c I_{h}+I_{m}$. From Model (7), then we have the following equations:

$$
\begin{aligned}
I^{\prime}= & \left(c I_{h}+I_{m}\right)^{\prime}=S_{h}\left[\frac{c \beta_{h} I_{h}}{S_{h}+I_{h}}+\frac{c \hat{\beta}_{m h} I_{m}}{S_{h}+I_{h}}+c \tilde{\beta}_{m h} I_{m}\right]-c \alpha I_{h}\left(S_{m}+I_{m}\right)-c\left(d_{h}+\mu_{h}\right) I_{h} \\
& +c \alpha\left[I_{h}\left(S_{m}+I_{m}\right)+S_{h} I_{m}\right]+\frac{\hat{\beta}_{h m} I_{h} S_{m}}{S_{h}+I_{h}}-\left(d_{m}+\mu_{m}\right) I_{m} \\
= & I_{h}\left[\frac{c \beta_{h} S_{h}}{N_{h}}+\frac{c \hat{\beta}_{h m} S_{m}}{N_{h}}\right]+I_{m}\left[\frac{c \hat{\beta}_{m h} S_{h}}{N_{h}}+c \tilde{\beta}_{m h}+c \alpha S_{h}\right]-c\left(d_{h}+\mu_{h}\right) I_{h}-\left(d_{m}+\mu_{m}\right) I_{m}
\end{aligned}
$$

This implies that

$$
I^{\prime} \geq I\left[\min \left\{\frac{\beta_{h} S_{h}}{N_{h}}+\frac{\hat{\beta}_{h m} S_{m}}{N_{h}}, \frac{c \hat{\beta}_{m h} S_{h}}{N_{h}}+c \tilde{\beta}_{m h}+c \alpha S_{h}\right\}-\max \left\{\left(d_{h}+\mu_{h}\right),\left(d_{m}+\mu_{m}\right)\right\}\right] .
$$

Assume that $\frac{r}{2 \sqrt{\hat{K}}}>d_{h}+\beta_{h}+\frac{\hat{\beta}_{m h} N^{*}}{\underline{N}_{h}^{*}}+\left(\tilde{\beta}_{m h}+\alpha\right)\left(N^{*}-\underline{N}_{h}^{*}\right)$ and $S_{h}(0)>S_{h}^{c}$, then according to Theorem 3.1 , we have

$$
\liminf _{t \rightarrow \infty} S_{h}(t) \geq S_{h}^{*}
$$




$$
\begin{aligned}
& \text { Therefore, if } \frac{\min \left\{\beta_{h}, c \hat{\beta}_{m h}+c \tilde{\beta}_{m h}+c \alpha S_{h}^{*}\right\}}{\max \left\{\left(d_{h}+\mu_{h}\right),\left(d_{m}+\mu_{m}\right)\right\}} \geq 1 \text {, then we have } \\
& \qquad \begin{aligned}
\left.\frac{I^{\prime}}{I}\right|_{I_{h}=I_{m}=0} & \geq\left.\left[\min \left\{\frac{\beta_{h} S_{h}}{N_{h}}, c \hat{\beta}_{m h}+c \tilde{\beta}_{m h}+c \alpha S_{h}\right\}-\max \left\{d_{h}+\mu_{h},\left(d_{m}+\mu_{m}\right)\right\}\right]\right|_{I_{h}=I_{m}=0} \\
& >\left[\min \left\{\beta_{h}, c \hat{\beta}_{m h}+c \tilde{\beta}_{m h}+c \alpha S_{h}^{*}\right\}-\max \left\{\left(d_{h}+\mu_{h}\right),\left(d_{m}+\mu_{m}\right)\right\}\right] \geq 0
\end{aligned}
\end{aligned}
$$

which implies that the virus $I$ persists by applying the average Lynapunov theorem (Hutson 1984).

On the other hand, we have the following inequalities:

$$
\begin{array}{ll}
I^{\prime} & \leq I\left[\max \left\{\frac{\beta_{h} S_{h}}{N_{h}}+\frac{\hat{\beta}_{h m} S_{m}}{N_{h}}, \frac{c \hat{\beta}_{m h} S_{h}}{N_{h}}+c \tilde{\beta}_{m h}+c \alpha S_{h}\right\}-\min \left\{\left(d_{h}+\mu_{h}\right),\left(d_{m}+\mu_{m}\right)\right\}\right] \\
\left.\frac{I^{\prime}}{I}\right|_{I_{h}=I_{m}=0}<\left[\max \left\{\beta_{h}+\frac{\hat{\beta}_{h m} S_{m}}{N_{h}}, \frac{c \hat{\beta}_{m h} S_{h}}{N_{h}}+c \tilde{\beta}_{m h}+c \alpha S_{h}\right\}-\min \left\{\left(d_{h}+\mu_{h}\right),\left(d_{m}+\mu_{m}\right)\right\}\right]
\end{array}
$$

Assume that $\frac{r}{2 \sqrt{\hat{K}}}>\frac{d_{h}+\mu_{h}+\alpha N^{*}}{\rho}, N_{h}(0)>\underline{N}_{h}^{c}$. Then according to Theorem 3.1, we have the following inequalities:

$$
\underline{N}_{h}^{*} \leq \liminf _{t \rightarrow \infty} N_{h}(t) \leq \limsup _{t \rightarrow \infty} N_{h}(t) \leq \bar{N}_{h}^{*} \leq \limsup _{t \rightarrow \infty} N(t) / c=N^{*} / c
$$

This implies the following inequality when $\frac{\max \left\{\beta_{h}+\frac{\hat{\beta}_{h m} N^{*}}{\underline{N}_{h}^{*}}, c \hat{\beta}_{m h}+c \tilde{\beta}_{m h}+c \alpha \bar{N}_{h}^{*}\right\}}{\min \left\{\left(d_{h}+\mu_{h}\right),\left(d_{m}+\mu_{m}\right)\right\}}<1$, then we have

$$
\left.\frac{I^{\prime}}{I}\right|_{I_{h}=I_{m}=0}<\left[\max \left\{\beta_{h}+\frac{\hat{\beta}_{h m} N^{*}}{\underline{\underline{N}}_{h}^{*}}, c \hat{\beta}_{m h}+c \tilde{\beta}_{m h}+c \alpha \bar{N}_{h}^{*}\right\}-\min \left\{\left(d_{h}+\mu_{h}\right),\left(d_{m}+\mu_{m}\right)\right\}\right]<0
$$

which implies that the virus goes extinct, i.e.,

$$
\lim _{t \rightarrow \infty} I(t)=0
$$

Proof of Theorem 3.3

Proof. If $M=0$, the virus-free subsystem (10) reduces to the only healthy honeybee population:

$$
S_{h}^{\prime}=\frac{r S_{h}^{2}}{\hat{K}+S_{h}^{2}}-d_{h} S_{h}
$$

which leads to the following three boundary equilibria if $\frac{r}{2 \hat{K}}>d_{h}$ :

$$
(0,0),\left(\bar{N}_{h}^{c}, 0\right), \text { and }\left(\bar{N}_{h}^{*}, 0\right) .
$$

The local stability can be easily determined by the eigenvalues evaluated at its Jacobian matrix. Simple algebraic calculations show that $\left(\bar{N}_{h}^{c}, 0\right)$ is a saddle if $\bar{N}_{h}^{c}<\frac{d_{m}}{\alpha c}$ while it is a source if $\bar{N}_{h}^{c}>\frac{d_{m}}{\alpha c}$; and $\left(\bar{N}_{h}^{*}, 0\right)$ is a sink if $\bar{N}_{h}^{*}<\frac{d_{m}}{\alpha c}$ while it is a saddle if $\bar{N}_{h}^{*}>\frac{d_{m}}{\alpha c}$.

Now let $(H, M)$ be an interior equilibrium of the virus-free subsystem (10), then we have the following 
equations hold:

$$
\begin{aligned}
& 0=c \alpha H M-d_{m} M \Leftrightarrow H=\frac{d_{m}}{c \alpha} \\
& 0=\frac{r H^{2}}{K+H^{2}}-d_{h} H-\alpha H M \Leftrightarrow M=\frac{1}{\alpha}\left(\frac{r H}{K+H^{2}}-d_{h}\right) .
\end{aligned}
$$

which gives the unique interior equilibrium $\left(H^{*}, M^{*}\right)=\left(\frac{d_{m}}{c \alpha}, \frac{1}{\alpha}\left(\frac{r H^{*}}{\hat{K}+\left(H^{*}\right)^{2}}-d_{h}\right)\right)$ provided that $\bar{N}_{h}^{c}<\frac{d_{m}}{\alpha c}<$ $\bar{N}_{h}^{*}$.

The local stability of $\left(H^{*}, M^{*}\right)$ is determined by the eigenvalues $\lambda_{i}, i=1,2$ of the following Jacobian matrix of (10):

$$
J_{H M}=\left(\begin{array}{cc}
\frac{r H^{*}\left(\hat{K}-\left(H^{*}\right)^{2}\right)}{\left(\hat{K}+\left(H^{*}\right)^{2}\right)^{2}} & -\alpha H^{*} \\
\alpha c M^{*} & 0
\end{array}\right)
$$

which gives the following two equations:

$$
\begin{aligned}
& \lambda_{1}+\lambda_{2}=\frac{r H^{*}\left(\hat{K}-\left(H^{*}\right)^{2}\right)}{\left(\hat{K}+\left(H^{*}\right)^{2}\right)^{2}} \\
& \lambda_{1} \lambda_{2}=c \alpha^{2} H^{*} M^{*}
\end{aligned} .
$$

Therefore, we can conclude that $\left(H^{*}, M^{*}\right)$ is a sink if $H^{*}>\sqrt{\hat{K}}$ while $\left(H^{*}, M^{*}\right)$ is a source if $H^{*}<\sqrt{\hat{K}}$. The discussion above implies follows:

1. If $\frac{r}{2 \sqrt{\hat{K}}}<d_{h}$, then the extinction equilibrium $(0,0)$ is the only locally stable equilibrium for the virus-free subsystem (10). Thus it is globally stable.

2. If $\frac{r}{2 \sqrt{\hat{K}}}>d_{h}$ and either $\bar{N}_{h}^{c}>\frac{d_{m}}{\alpha c}$ or $\frac{d_{m}}{\alpha c}>\bar{N}_{h}^{*}$, then the virus-free subsystem (10) has three boundary equilibria: $(0,0),\left(S_{h}^{c}, 0\right),\left(\bar{N}_{h}^{*}, 0\right)$ where $(0,0)$ is always locally stable.

- If $\bar{N}_{h}^{c}>\frac{d_{m}}{\alpha c}$, then $\left(S_{h}^{c}, 0\right)$ is a source and $\left(\bar{N}_{h}^{*}, 0\right)$ is a saddle. Since (10) is a two-D ode system, it has global stability at the extinction equilibrium $(0,0)$ according to the Poincare-Bendison theorem [49].

- If $\bar{N}_{h}^{*}<\frac{d_{m}}{\alpha c}$, then $\left(S_{h}^{c}, 0\right)$ is a saddle and $\left(\bar{N}_{h}^{*}, 0\right)$ is a sink. This implies that (10) has two locally asymptotically stable boundary equilibria $(0,0)$ and $\left(\bar{N}_{h}^{*}, 0\right)$ which are reserved as the only two attractors for the model.

3. If $\frac{r}{2 \sqrt{\hat{K}}}>d_{h}$ and $\bar{N}_{h}^{c}<\frac{d_{m}}{\alpha c}<\bar{N}_{h}^{*}$, then (10) has three boundary equilibria $(0,0),\left(S_{h}^{c}, 0\right),\left(\bar{N}_{h}^{*}, 0\right)$ and the unique interior equilibrium $\left(H^{*}, M^{*}\right)$ where $(0,0)$ is locally stable, both $\left(S_{h}^{c}, 0\right)$ and $\left(\bar{N}_{h}^{*}, 0\right)$ are saddle nodes. The local stability of $\left(H^{*}, M^{*}\right)$ is determined by the sign of $H^{*}-\sqrt{\hat{K}}$ : if $H^{*}-\sqrt{\hat{K}}>0$, then $\left(H^{*}, M^{*}\right)$ is locally asymptotically stable while if $H^{*}-\sqrt{\hat{K}}<0$, then $\left(H^{*}, M^{*}\right)$ is a source.

Define $\mathcal{R}_{0}^{M}=\frac{\bar{N}_{h}^{*}}{H^{*}}$. Then we can conclude that (10) has no interior equilibrium if $\mathcal{R}_{0}^{M}<1$ or $\mathcal{R}_{0}^{M}>\frac{\bar{N}_{h}^{*}}{S_{h}^{c}}>1$; and (10) has a unique interior equilibrium $\left(H^{*}, M^{*}\right)$ if $1<\mathcal{R}_{0}^{M}<\frac{\bar{N}_{h}^{*}}{S_{h}^{c}}$. More specifically, $\left(H^{*}, M^{*}\right)$ is locally stable if $1<\mathcal{R}_{0}^{M}<\frac{\bar{N}_{h}^{*}}{\bar{K}}$ while it is a source if $\mathcal{R}_{0}^{M}>\max \left\{1, \frac{\bar{N}_{h}^{*}}{\bar{K}}\right\}$. 
Proof of Theorem 3.4

Proof. If $I=0$, the mite-free subsystem (8) reduces to the only healthy honeybee population:

$$
S_{h}^{\prime}=\frac{r S_{h}^{2}}{\hat{K}+S_{h}^{2}}-d_{h} S_{h}
$$

which leads to the following three boundary equilibria if $\frac{r}{2 \widehat{K}}>d_{h}$ :

$$
(0,0),\left(\bar{N}_{h}^{c}, 0\right) \text {, and }\left(\bar{N}_{h}^{*}, 0\right) \text {. }
$$

The local stability can be easily determined by the eigenvalues evaluated at its Jacobian matrix. Define $\mathcal{R}_{0}^{V}=\frac{\beta_{h}}{d_{h}+\mu_{h}}$. The simple algebraic calculations show that if $\mathcal{R}_{0}^{V}<1,\left(\bar{N}_{h}^{c}, 0\right)$ is a saddle and $\left(\bar{N}_{h}^{*}, 0\right)$ is a sink; while if $\mathcal{R}_{0}^{V}>1$, then $\left(\bar{N}_{h}^{c}, 0\right)$ is a source and $\left(\bar{N}_{h}^{*}, 0\right)$ is a saddle. According to Theorem 3.1, the extinction equilibrium $(0,0)$ is always a sink.

Let $(S, I)$ be an interior equilibrium of the mite-free subsystem (8), then it satisfies the following equations:

$$
\begin{aligned}
\frac{\beta_{h} S}{S+I}-\left(d_{h}+\mu_{h}\right)=0 & \Rightarrow S=\frac{d_{h}+\mu_{h}}{\beta_{h}-\left(d_{h}+\mu_{h}\right)} I=\frac{I}{\frac{\beta_{h}}{d_{h}+\mu_{h}}-1}=a I \\
\frac{r(S+\rho I)^{2}}{\hat{K}+(S+\rho I)^{2}}-d_{h} S-S \frac{\beta_{h} I}{S+I}=0 & \Rightarrow \frac{r I}{\frac{K}{(a+\rho)^{2}}+I^{2}}-a\left(d_{h}+\frac{\beta_{h}}{1+a}\right)=0 \\
\frac{r(S+\rho I)^{2}}{\hat{K}+(S+\rho I)^{2}}-d_{h} S-S \frac{\beta_{h} I}{S+I}=0 & \Rightarrow \frac{r(S+\rho I)^{2}}{\hat{K}+(S+\rho I)^{2}}-d_{h} S-\left(d_{h}+\mu_{h}\right) I=0 \\
& \Rightarrow \frac{r I}{\frac{K}{(a+\rho)^{2}}+I^{2}}-\left((a+1) d_{h}+\mu_{h}\right)=\frac{r I}{\frac{K}{(a+\rho)^{2}}+I^{2}}-\tilde{d}=0 \\
& \Rightarrow \frac{a \beta_{h}}{1+a}=d_{h}+\mu_{h} .
\end{aligned}
$$

Therefore, if $\mathcal{R}_{0}^{V}=\frac{\beta_{h}}{d_{h}+\mu_{h}}>1$ and $\frac{r}{d}>\frac{2 \sqrt{\hat{K}}}{a+\rho}$, then we can conclude that the mite-free subsystem (8) can have two interior equilibria $\left(S_{h}^{k}, I_{h}^{k}\right), k=1,2$ where

$$
a=\frac{1}{\frac{\beta_{h}}{d_{h}+\mu_{h}}-1}, d=(a+1) d_{h}+\mu_{h}=d_{h}\left(\frac{\frac{\beta_{h}}{d_{h}+\mu_{h}}}{\frac{\beta_{h}}{d_{h}+\mu_{h}}-1}\right)+\mu_{h},
$$

and

$$
I_{h}^{1}=\frac{\frac{r}{d}-\sqrt{\left(\frac{r}{d}\right)^{2}-4 \frac{\hat{K}}{(a+\rho)^{2}}}}{2}, I_{h}^{2}=\frac{\frac{r}{d}+\sqrt{\left(\frac{r}{d}\right)^{2}-4 \frac{\hat{K}}{(a+\rho)^{2}}}}{2}, S_{h}^{k}=a I_{h}^{k}, k=1,2 .
$$

Now we examine the local stability of these two interior equilibria provided that they exist. The Jacobian matrix of (8) evaluated at the interior equilibrium $(S, I)=(a I, I)$ can be expressed as follows:

$$
J_{H V}=\left(\begin{array}{cc}
\frac{2 r \hat{K}(a+\rho) I}{\left((a+\rho)^{2} I^{2}+\hat{K}\right)^{2}}-d_{h}-\frac{\beta_{h}}{(1+a)^{2}} & \frac{2 r \rho \hat{K}(a+\rho) I}{\left((a+\rho)^{2} I^{2}+\hat{K}\right)^{2}}-\frac{a^{2} \beta_{h}}{(1+a)^{2}} \\
\frac{\beta_{h}}{(1+a)^{2}} & -\frac{a \beta_{h}}{(1+a)^{2}}
\end{array}\right)
$$


whose two eigenvalues $\lambda_{i}, i=1,2$ satisfy the following equations:

$$
\begin{aligned}
\lambda_{1}+\lambda_{2} & =\frac{2 r \hat{K}(a+\rho) I}{\left((a+\rho)^{2} I^{2}+\hat{K}\right)^{2}}-d_{h}-\frac{\beta_{h}}{1+a}=\frac{2 \hat{K}}{\left((a+\rho)^{2} I^{2}+\hat{K}\right)(a+\rho)} \frac{r(a+\rho)^{2} I}{(a+\rho)^{2} I^{2}+\hat{K}}-d_{h}-\frac{\beta_{h}}{1+a} \\
& =\frac{2 \hat{K}}{\left((a+\rho)^{2} I^{2}+\hat{K}\right)(a+\rho)} a\left(d_{h}+\frac{\beta_{h}}{1+a}\right)-d_{h}-\frac{\beta_{h}}{1+a}=\left(d_{h}+\frac{\beta_{h}}{1+a}\right)\left[\frac{2 a \hat{K}}{\left((a+\rho)^{2} I^{2}+\hat{K}\right)(a+\rho)}-1\right] \\
& <\left(d_{h}+\frac{\beta_{h}}{1+a}\right)\left[\frac{2 \hat{K}}{(a+\rho)^{2} I^{2}+\hat{K}}-1\right]
\end{aligned}
$$

and

$$
\begin{aligned}
\lambda_{1} \lambda_{2} & =\frac{\beta_{h}}{(1+a)^{2}}\left[-\frac{2 r a \hat{K}(a+\rho) I}{\left((a+\rho)^{2} I^{2}+\hat{K}\right)^{2}}+a d_{h}+\frac{a \beta_{h}}{(1+a)^{2}}-\frac{2 r \rho \hat{K}(a+\rho) I}{\left((a+\rho)^{2} I^{2}+\hat{K}\right)^{2}}+\frac{a^{2} \beta_{h}}{(1+a)^{2}}\right] \\
& =\frac{\beta_{h}}{(1+a)^{2}}\left[-\frac{2 r \hat{K}(a+\rho)^{2} I}{\left((a+\rho)^{2} I^{2}+\hat{K}\right)^{2}}+a d_{h}+\frac{a \beta_{h}}{1+a}\right]=\frac{\beta_{h}}{(1+a)^{2}}\left[-\frac{2 r \hat{K}(a+\rho)^{2} I}{\left((a+\rho)^{2} I^{2}+\hat{K}\right)^{2}}+\tilde{d}\right] .
\end{aligned}
$$

If $\mathcal{R}_{0}^{V}=\frac{\beta_{h}}{d_{h}+\mu_{h}}>1$ and $\frac{r}{d}>\frac{2 \sqrt{\hat{K}}}{a+\rho}$, then we have two interior equilibria $\left(S_{h}^{k}, I_{h}^{k}\right), k=1,2$ where

$$
I_{h}^{1}=\frac{\frac{r}{\tilde{d}}-\sqrt{\left(\frac{r}{\tilde{d}}\right)^{2}-4 \frac{\hat{K}}{(a+\rho)^{2}}}}{2}, I_{h}^{2}=\frac{\frac{r}{\tilde{d}}+\sqrt{\left(\frac{r}{\tilde{d}}\right)^{2}-4 \frac{\hat{K}}{(a+\rho)^{2}}}}{2}, S_{h}^{k}=a I_{h}^{k}, k=1,2 .
$$

This implies that $\lambda_{1}\left(I_{h}^{2}\right) \lambda_{2}\left(I_{h}^{1}\right)<0, \lambda_{1}\left(I_{h}^{2}\right) \lambda_{2}\left(I_{h}^{2}\right)>0$ and

$$
\begin{aligned}
\lambda_{1}\left(I_{h}^{2}\right)+\lambda_{2}\left(I_{h}^{2}\right) & <\left(d_{h}+\frac{\beta_{h}}{1+a}\right)\left[\frac{2 \hat{K}}{(a+\rho)^{2} I^{2}+\hat{K}}-1\right] \\
& <\left(d_{h}+\frac{\beta_{h}}{1+a}\right)\left[\frac{2 \hat{K}}{(a+\rho)^{2} \frac{\hat{K}}{(a+\rho)^{2}}+\hat{K}}-1\right]=0
\end{aligned}
$$

since

$$
I_{h}^{2}=\frac{\frac{r}{\tilde{d}}+\sqrt{\left(\frac{r}{\tilde{d}}\right)^{2}-4 \frac{\hat{K}}{(a+\rho)^{2}}}}{2}>\frac{r}{2 \tilde{d}}>\frac{\sqrt{\hat{K}}}{(a+\rho)} \Rightarrow\left(I_{h}^{2}\right)^{2}>\frac{\hat{K}}{(a+\rho)^{2}} .
$$

Therefore, we can conclude that if $\mathcal{R}_{0}^{V}=\frac{\beta_{h}}{d_{h}+\mu_{h}}>1$ and $\frac{r}{d}>\frac{2 \sqrt{\hat{K}}}{a+\rho}$, then the system has two interior equilibria $\left(S_{h}^{k}, I_{h}^{k}\right), k=1,2$ where $\left(S_{h}^{1}, I_{h}^{1}\right)$ is always a saddle while $\left(S_{h}^{2}, I_{h}^{2}\right)$ is always locally asymptotically stable.

Notice that $\frac{r}{d}>\frac{2 \sqrt{\hat{K}}}{a+\rho} \Leftrightarrow \frac{r}{2 \sqrt{\hat{K}}}>\frac{\tilde{d}}{a+\rho}=\frac{d_{h}(a+1)}{a+\rho}+\frac{\mu_{h}}{a+\rho}>d_{h}$. The discussions above show that the mite-free subsystem (8) has no interior equilibrium if either $\mathcal{R}_{0}^{V}<1$ or $\frac{r}{2 \sqrt{\hat{K}}}<\frac{\tilde{d}}{a+\rho}$ holds. This leads to the following two cases:

1. If either $\frac{r}{2 \sqrt{\hat{K}}}<d_{h}$, then the mite-free subsystem (8) has only the extinction equilibrium $(0,0)$ which is locally stable, thus it is globally stable.

2. If $\mathcal{R}_{0}^{V}>1$ and $d_{h}<\frac{r}{2 \sqrt{\hat{K}}}<\frac{\tilde{d}}{a+\rho}$, then the mite-free subsystem (8) has the following three boundary equilibria

$$
(0,0),\left(\bar{N}_{h}^{c}, 0\right), \text { and }\left(\bar{N}_{h}^{*}, 0\right)
$$

where $(0,0)$ is locally asymptotically stable; $\left(\bar{N}_{h}^{c}, 0\right)$ is a source; and $\left(\bar{N}_{h}^{*}, 0\right)$ is a saddle. Then according to the Poincare-Bendison theorem, we can conclude that all interior points of $\mathbb{R}_{+}^{2}$ converges to the 
extinction equilibrium $(0,0)$.

3. If $\mathcal{R}_{0}^{V}<1$ and $d_{h}<\frac{r}{2 \sqrt{\hat{K}}}$, then the mite-free subsystem (8) has the following three boundary equilibria

$$
(0,0),\left(\bar{N}_{h}^{c}, 0\right), \text { and }\left(\bar{N}_{h}^{*}, 0\right)
$$

where $(0,0)$ is locally asymptotically stable; $\left(\bar{N}_{h}^{c}, 0\right)$ is a saddle; and $\left(\bar{N}_{h}^{*}, 0\right)$ is a sink. This implies that both $(0,0)$, and $\left(\bar{N}_{h}^{*}, 0\right)$ are locally asymptotically stable.

Proof of Theorem 3.5

Proof. Let $N_{h}=S_{h}+I_{h}$, then from Model (9), we obtain

$$
\begin{aligned}
N_{h}^{\prime} & =\frac{r\left(S_{h}+\rho I_{h}\right)^{2}}{\hat{K}+\left(S_{h}+\rho I_{h}\right)^{2}}-d_{h} N_{h}-\mu_{h} I_{h} \\
& \leq \frac{r N_{h}^{2}}{\hat{K}+N_{h}^{2}}-d_{h} N_{h}
\end{aligned}
$$

which implies that when $\frac{r}{2 \sqrt{\hat{K}}}>d_{h}$, we have

$$
\limsup _{t \rightarrow \infty}\left(S_{h}(t)+I_{h}(t)\right)=\limsup _{t \rightarrow \infty} N_{h}(t) \leq \frac{\frac{r}{d_{h}}+\sqrt{\left(\frac{r}{d_{h}}\right)^{2}-4 \hat{K}}}{2}=\bar{N}_{h}^{*} .
$$

Therefore, we can conclude that if the inequalities $\frac{r}{d_{h}}>2 \sqrt{\hat{K}}$ and $\bar{N}_{h}^{*}<d_{m}+\mu_{m}$ hold, then we have $\lim \sup _{t \rightarrow \infty} S_{m}(t)=0$ since

$$
I_{m}^{\prime}=c \alpha I_{m}\left[I_{h}+S_{h}-\frac{\left(d_{m}+\mu_{m}\right)}{c \alpha}\right]=c \alpha I_{m}\left[N_{h}-\frac{\left(d_{m}+\mu_{m}\right)}{c \alpha}\right] \leq c \alpha I_{m}\left[\bar{N}_{h}^{*}-\frac{\left(d_{m}+\mu_{m}\right)}{c \alpha}\right]<0 .
$$

This implies that the limiting dynamics of Model (9) is reduced to the mite-free model (8).

An interior equilibrium $\left(S_{h}, I_{h}, I_{m}\right)$ of the healthy-mite-free subsystem (9), satisfies the following equations:

$$
\begin{aligned}
c \alpha I_{m}\left[I_{h}+S_{h}-\frac{d_{m}+\mu_{m}}{c \alpha}\right] & =0 \Rightarrow N_{h}=I_{h}+S_{h}=\frac{d_{m}+\mu_{m}}{c \alpha} \\
\frac{r\left(S_{h}+\rho I_{h}\right)^{2}}{\hat{K}+\left(S_{h}+\rho I_{h}\right)^{2}}-d_{h} N_{h}-\alpha N_{h} I_{m}-\mu_{h} I_{h} & =0 \Rightarrow \frac{r\left(S_{h}+\rho I_{h}\right)^{2}}{\hat{K}+\left(S_{h}+\rho I_{h}\right)^{2}}-d_{h} N_{h}-\alpha N_{h} I_{m}-\mu_{h} I_{h}=0 \\
& \Rightarrow \quad \frac{r\left(N_{h}-I_{h}+\rho I_{h}\right)^{2}}{\hat{K}+\left(N_{h}-I_{h}+\rho I_{h}\right)^{2}}-d_{h} N_{h}-\alpha N_{h} I_{m}-\mu_{h} I_{h}=0 \\
& \Rightarrow I_{m}=\frac{\frac{r\left(\frac{d_{m}+\mu_{m}}{c \alpha}-I_{h}+\rho I_{h}\right)^{2}}{\hat{K}+\left(\frac{d_{m}+\mu_{m}}{c \alpha}-I_{h}+\rho I_{h}\right)^{2}}-d_{h} \frac{d_{m}+\mu_{m}}{c \alpha}-\mu_{h} I_{h}}{\alpha\left(\frac{d_{m}+\mu_{m}}{c \alpha}\right)}=f_{1}\left(I_{h}\right)
\end{aligned}
$$




$$
\begin{aligned}
S_{h}\left[\frac{\beta_{h} I_{h}}{S_{h}+I_{h}}+\frac{\hat{\beta}_{m h} I_{m}}{S_{h}+I_{h}}+\tilde{\beta}_{m h} I_{m}\right] & =\alpha I_{h} I_{m}+\left(d_{h}+\mu_{h}\right) I_{h} \\
\left(\frac{d_{m}+\mu_{m}}{c \alpha}-I_{h}\right)\left[\frac{\beta_{h} I_{h}}{d_{m}+\mu_{m}}+\frac{c \alpha \hat{\beta}_{m h} I_{m}}{d_{m}+\mu_{m}}+\tilde{\beta}_{m h} I_{m}\right] & =\alpha I_{h} I_{m}+\left(d_{h}+\mu_{h}\right) I_{h} \\
& \Rightarrow I_{m}=\frac{\left(\frac{d_{m}+\mu_{m}}{c \alpha}-I_{h}\right)\left[\frac{\beta_{h}}{d I_{m}}-\left(d_{h}+\mu_{h}\right)\right] I_{h}}{\left.\alpha \frac{d_{m}+\mu_{m}}{c \alpha}-I_{h}\right)\left(\frac{c \alpha \hat{\beta}_{m h}}{d_{m}+\mu_{m}}+\tilde{\beta}_{m h}\right)} \\
& \Rightarrow I_{m}=\frac{\left(\frac{d_{m}+\mu_{m}}{c \alpha}-I_{h}\right)\left[\frac{\beta_{h}}{d_{m}+\mu_{m}}-\left(d_{h}+\mu_{h}\right)\right] I_{h}}{\left[\alpha+\frac{c \alpha \hat{\beta}_{m h}}{d_{m}+\mu_{m}}+\tilde{\beta}_{m h}\right] I_{h}-\frac{d_{m}+\mu_{m}}{c \alpha}\left(\frac{c \alpha \hat{\beta}_{m h}}{d_{m}+\mu_{m}}+\tilde{\beta}_{m h}\right)}=f_{2}\left(I_{h}\right)
\end{aligned}
$$

The equations above imply that the interior equilibrium of (9) is the positive intercept of $f_{1}\left(I_{h}\right)$ and $f_{2}\left(I_{h}\right)$ subject to $0<I_{h}<\frac{d_{m}+\mu_{m}}{c \alpha}$. The expression for the function $f_{1}\left(I_{h}\right)$ implies that the subsystem (9) has no interior equilibrium if

$$
r<\frac{d_{h}\left(d_{m}+\mu_{m}\right)}{c \alpha} \Leftrightarrow r c \alpha<d_{h}\left(d_{m}+\mu_{m}\right)
$$

since $I_{m}=f_{1}\left(I_{h}\right)<0$ when this inequality holds.

Assume that $\frac{r}{2 \sqrt{\hat{K}}}>\frac{d_{h}+\mu_{h}+\alpha N^{*}}{\rho}$ and $N_{h}(0)>\underline{N}_{h}^{c}$, then according to Theorem 3.1 and 3.2 , then we have

$$
\underline{N}_{h}^{c}<\underline{N}_{h}^{*} \leq \liminf _{t \rightarrow \infty} N_{h}(t) \leq \limsup _{t \rightarrow \infty} N_{h}(t) \leq \bar{N}_{h}^{*}
$$

which implies that the set $\left\{S_{h}+I_{h} \geq \underline{N}_{h}^{c}\right\}$ is invariant. If $I_{m}=0$, then the subsystem (9) reduces to the mite-free system (8). According to Theorem 3.4, the omega limit set of the mite-free system $(8)$ is $\left(\bar{N}_{h}^{*}, 0\right)$ when $\mathcal{R}_{0}^{V}=\frac{\beta_{h}}{d_{h}+\mu_{h}}<1$ holds. If $\bar{N}_{h}^{*}>\frac{d_{m}+\mu_{m}}{c \alpha}$, then we have

$$
\left.\frac{I_{m}^{\prime}}{I_{m}}\right|_{I_{m}=0}=c \alpha\left(\bar{N}_{h}^{*}-\frac{d_{m}+\mu_{m}}{c \alpha}\right)>0
$$

This implies that the virus $I_{m}$ persists by applying the average Lynapunov theorem (Hutson 1984). Notice that

$$
\left.I_{h}\right|_{I_{h}=0}=\frac{\hat{\beta}_{m h} S_{h} I_{m}}{N_{h}}>0 .
$$

Therefore, the virus infected honeybees $I_{h}$ also persists.

\section{Proof of Theorem 3.6}

Proof. The first part of Theorem 3.6 can be deduced directly from Theorem 3.1, 3.2, 3.3, 3.4, and 3.5. We focus on the sufficient conditions that lead to no interior equilibrium (Item 3) and the persistence of mites (Item 4).

$$
\begin{aligned}
& \text { If }\left(S_{h}, I_{h}, S_{m}, I_{m}\right) \text { is an interior equilibrium of the system }(7) \text {, then it satisfies the following equations: } \\
& \qquad \begin{aligned}
0 & =S_{m}\left[c \alpha S_{h}-\frac{\hat{\beta}_{h m} I_{h}}{S_{h}+I_{h}}-d_{m}\right] \\
& \Rightarrow I_{h}=S_{h} \frac{c \alpha S_{h}-d_{m}}{d_{m}+\hat{\beta}_{h m}-c \alpha S_{h}}, \frac{d_{m}}{c \alpha}<S_{h}<\frac{d_{m}+\hat{\beta}_{h m}}{c \alpha} \\
\Rightarrow & N_{h}=S_{h}+I_{h}=\frac{\hat{\beta}_{h m} S_{h}}{d_{m}+\hat{\beta}_{h m}-c \alpha S_{h}}=g_{1}\left(S_{h}\right)
\end{aligned}
\end{aligned}
$$


Therefore, the interior equilibrium $\left(S_{h}, I_{h}, S_{m}, I_{m}\right)$ are positive solutions of the following four equations:

$$
\begin{aligned}
N_{h} & =S_{h}+I_{h}=g_{1}\left(S_{h}\right)=\frac{\hat{\beta}_{h m} S_{h}}{d_{m}+\hat{\beta}_{h m}-c \alpha S_{h}} \text { subject to } S_{h}<\frac{d_{m}+\hat{\beta}_{h m}}{c \alpha} \\
\frac{I_{m}}{N_{m}} & =g_{3}\left(S_{h}\right)=\frac{\left(c \alpha S_{h}-d_{m}\right)\left(\hat{\beta}_{h m}+d_{m}\right)}{\mu_{m}\left(\hat{\beta}_{h m}+d_{m}-c \alpha S_{h}\right)}<1 \text { subject to } \frac{d_{m}}{c \alpha}<S_{h}<\frac{d_{m}+\hat{\beta}_{h m}}{c \alpha} \frac{d_{m}+\mu_{m}}{d_{m}+\hat{\beta}_{h m}+\mu_{m}} \\
N_{m} & =g_{2}\left(S_{h}\right)=\frac{r S_{h}\left(\hat{\beta}_{h m}+\left(d_{m}-c \alpha S_{h}\right)\right)\left(\hat{\beta}_{h m}+\left(d_{m}-c \alpha S_{h}\right)(1-\rho)\right)^{2}}{\alpha \hat{\beta}_{h m}\left[\hat{K}\left(\hat{\beta}_{h m}+\left(d_{m}-c \alpha S_{h}\right)\right)^{2}+S_{h}^{2}\left(\hat{\beta}_{h m}+\left(d_{m}-c \alpha S_{h}\right)(1-\rho)\right)^{2}\right]}-\frac{d_{h}}{\alpha}-\frac{\mu_{h}\left(c \alpha S_{h}-d_{m}\right)}{\hat{\beta}_{h m}} \\
N_{m} & =g_{4}\left(S_{h}\right)=\frac{\frac{\beta_{h}\left(d_{m}+\hat{\beta}_{h m}-c \alpha S_{h}\right)}{\hat{\beta}_{h m}-\left(d_{h}+\mu_{h}\right)}}{\alpha-\frac{\hat{\beta}_{m h}\left(\hat{\beta}_{h m}+d_{m}\right)\left(d_{m}+\hat{\beta}_{h m}-c \alpha S_{h}\right)}{\mu_{m} \hat{\beta}_{h m} S_{h}}-\frac{\hat{\beta}_{m h}\left(\hat{\beta}_{h m}+d_{m}\right)}{\mu_{m}}} \\
& =\frac{\mu_{m} S_{h}\left[\beta_{h}\left(d_{m}+\hat{\beta}_{h m}\right)-\hat{\beta}_{h m}\left(d_{h}+\mu_{h}\right)-c \alpha \beta_{h} S_{h}\right]}{\left[\alpha \mu_{m} \hat{\beta}_{h m}+c \alpha \hat{\beta}_{m h}\left(\hat{\beta}_{h m}+d_{m}\right)-\hat{\beta}_{m h} \hat{\beta}_{h m}\left(\hat{\beta}_{h m}+d_{m}\right)\right] S_{h}-\hat{\beta}_{m h}\left(\hat{\beta}_{h m}+d_{m}\right)\left(d_{m}+\hat{\beta}_{h m}\right)}
\end{aligned}
$$

Thus, we can conclude the following statements regarding the sign of $g_{4}\left(S_{h}\right)$ :

1. If $\beta_{h}\left(d_{m}+\hat{\beta}_{h m}\right)<\hat{\beta}_{h m}\left(d_{h}+\mu_{h}\right)$ and $\alpha \mu_{m} \hat{\beta}_{h m}+c \alpha \hat{\beta}_{m h}\left(\hat{\beta}_{h m}+d_{m}\right)<\tilde{\beta}_{m h} \hat{\beta}_{h m}\left(\hat{\beta}_{h m}+d_{m}\right)$, then $g_{4}\left(S_{h}\right)>0$ for all $S_{h}>0$.

2. If $\beta_{h}\left(d_{m}+\hat{\beta}_{h m}\right)>\hat{\beta}_{h m}\left(d_{h}+\mu_{h}\right)$ and $\alpha \mu_{m} \hat{\beta}_{h m}+c \alpha \hat{\beta}_{m h}\left(\hat{\beta}_{h m}+d_{m}\right)>\tilde{\beta}_{m h} \hat{\beta}_{h m}\left(\hat{\beta}_{h m}+d_{m}\right)$, then $g_{4}\left(S_{h}\right)>0$ when

$$
\frac{\hat{\beta}_{m h}\left(\hat{\beta}_{h m}+d_{m}\right)\left(d_{m}+\hat{\beta}_{h m}\right)}{\alpha \mu_{m} \hat{\beta}_{h m}+c \alpha \hat{\beta}_{m h}\left(\hat{\beta}_{h m}+d_{m}\right)-\tilde{\beta}_{m h} \hat{\beta}_{h m}\left(\hat{\beta}_{h m}+d_{m}\right)}<S_{h}<\frac{\beta_{h}\left(d_{m}+\hat{\beta}_{h m}\right)-\hat{\beta}_{h m}\left(d_{h}+\mu_{h}\right)}{c \alpha \beta_{h}} .
$$


3. If $\beta_{h}\left(d_{m}+\hat{\beta}_{h m}\right)<\hat{\beta}_{h m}\left(d_{h}+\mu_{h}\right)$ and $\alpha \mu_{m} \hat{\beta}_{h m}+c \alpha \hat{\beta}_{m h}\left(\hat{\beta}_{h m}+d_{m}\right)>\tilde{\beta}_{m h} \hat{\beta}_{h m}\left(\hat{\beta}_{h m}+d_{m}\right)$, then $g_{4}\left(S_{h}\right)>0$ when

$$
S_{h}<\frac{\hat{\beta}_{m h}\left(\hat{\beta}_{h m}+d_{m}\right)\left(d_{m}+\hat{\beta}_{h m}\right)}{\alpha \mu_{m} \hat{\beta}_{h m}+c \alpha \hat{\beta}_{m h}\left(\hat{\beta}_{h m}+d_{m}\right)-\tilde{\beta}_{m h} \hat{\beta}_{h m}\left(\hat{\beta}_{h m}+d_{m}\right)} .
$$

4. If $\beta_{h}\left(d_{m}+\hat{\beta}_{h m}\right)>\hat{\beta}_{h m}\left(d_{h}+\mu_{h}\right)$ and $\alpha \mu_{m} \hat{\beta}_{h m}+c \alpha \hat{\beta}_{m h}\left(\hat{\beta}_{h m}+d_{m}\right)<\tilde{\beta}_{m h} \hat{\beta}_{h m}\left(\hat{\beta}_{h m}+d_{m}\right)$, then $g_{4}\left(S_{h}\right)>0$ when

$$
S_{h}>\frac{\beta_{h}\left(d_{m}+\hat{\beta}_{h m}\right)-\hat{\beta}_{h m}\left(d_{h}+\mu_{h}\right)}{c \alpha \beta_{h}} .
$$

Notice that the interior equilibrium $\left(S_{h}, I_{h}, S_{m}, I_{m}\right)$ requires $g_{3}\left(S_{h}\right)>0$, i.e., $\frac{d_{m}}{c \alpha}<S_{h}<\frac{d_{m}+\hat{\beta}_{h m}}{c \alpha} \frac{d_{m}+\mu_{m}}{d_{m}+\hat{\beta}_{h m}+\mu_{m}}$. Therefore, the interior equilibrium $\left(S_{h}, I_{h}, S_{m}, I_{m}\right)$ does not exist if one of the following inequalities hold

1. $\frac{\beta_{h}}{d_{h}+\mu_{h}}>\frac{\hat{\beta}_{h m}}{d_{m}+\hat{\beta}_{h m}}, \frac{\alpha \mu_{m} \hat{\beta}_{h m}}{\hat{\beta}_{h m}+d_{m}}>\tilde{\beta}_{m h} \hat{\beta}_{h m}-c \alpha$ and

$$
\frac{\hat{\beta}_{m h}}{\frac{\alpha \mu_{m} \hat{\beta}_{h m}}{\left(d_{m}+\hat{\beta}_{h m}\right)}+c \alpha \hat{\beta}_{m h}-\tilde{\beta}_{m h} \hat{\beta}_{h m}}>\frac{d_{m}+\mu_{m}}{c \alpha\left(d_{m}+\hat{\beta}_{h m}+\mu_{m}\right)} \text { or } \frac{\frac{\beta_{h}}{d_{h}+\mu_{h}}-\frac{\hat{\beta}_{h m}}{d_{m}+\hat{\beta}_{h m}}}{\beta_{h}\left(d_{m}+\hat{\beta}_{h m}\right)\left(d_{h}+\mu_{h}\right)}<d_{m} .
$$

2. $\frac{\beta_{h}}{d_{h}+\mu_{h}}<\frac{\hat{\beta}_{h m}}{d_{m}+\hat{\beta}_{h m}}, \frac{\alpha \mu_{m} \hat{\beta}_{h m}}{\hat{\beta}_{h m}+d_{m}}>\tilde{\beta}_{m h} \hat{\beta}_{h m}-c \alpha$ and $\frac{\hat{\beta}_{m h}\left(d_{m}+\hat{\beta}_{h m}\right)}{\frac{\alpha \mu_{m} \hat{\beta}_{h m}}{\left(d_{m}+\hat{\beta}_{h m}\right)}+c \alpha \hat{\beta}_{m h}-\tilde{\beta}_{m h} \hat{\beta}_{h m}}<\frac{d_{m}}{c \alpha}$.

3. $\frac{\beta_{h}}{d_{h}+\mu_{h}}>\frac{\hat{\beta}_{h m}}{d_{m}+\hat{\beta}_{h m}}, \frac{\alpha \mu_{m} \hat{\beta}_{h m}}{\hat{\beta}_{h m}+d_{m}}<\tilde{\beta}_{m h} \hat{\beta}_{h m}-c \alpha$ and $\frac{\frac{\beta_{h}}{d_{h}+\mu_{h}}-\frac{\hat{\beta}_{h m}}{d_{m}+\hat{\beta}_{h m}}}{\beta_{h}\left(d_{m}+\hat{\beta}_{h m}\right)\left(d_{h}+\mu_{h}\right)}>\frac{\left(d_{m}+\mu_{m}\right)\left(d_{m}+\hat{\beta}_{h m}\right)}{d_{m}+\hat{\beta}_{h m}+\mu_{m}}$.

Assume that $\frac{r}{2 \sqrt{\hat{K}}}>\frac{d_{h}+\mu_{h}+\alpha N^{*}}{\rho}$ and $N_{h}(0)>\underline{N}_{h}^{c}$, then according to Theorem 3.1 and 3.2 , then we have

$$
\underline{N}_{h}^{c}<\underline{N}_{h}^{*} \leq \liminf _{t \rightarrow \infty} N_{h}(t) \leq \limsup _{t \rightarrow \infty} N_{h}(t) \leq \bar{N}_{h}^{*}
$$

which implies that the set $\left\{S_{h}+I_{h} \geq \underline{N}_{h}^{c}\right\}$ is invariant. If $S_{m}=I_{m}=0$, then the full system (7) reduces to the mite-free system (8). According to Theorem 3.4, the omega limit set of the mite-free system (8) is $\left(\bar{N}_{h}^{*}, 0\right)$ when $\mathcal{R}_{0}^{V}=\frac{\beta_{h}}{d_{h}+\mu_{h}}<1$ holds. If $\bar{N}_{h}^{*}>\frac{d_{m}+\mu_{m}}{c \alpha}$, then we have

$$
\left.\frac{N_{m}^{\prime}}{N_{m}}\right|_{N_{m}=0} \geq c \alpha\left(\bar{N}_{h}^{*}-\frac{d_{m}+\mu_{m}}{c \alpha}\right)>0 .
$$

This implies that the mite population $N_{m}$ persists by applying the average Lynapunov theorem (Hutson 1984).

\section{Acknowledgements}

Y.K's research is partially supported by NSF-DMS (1313312). We really appreciate useful comments and suggestions provided by two reviewers to significantly improve this manuscript. 


\section{References}

References

[1] R. M. Anderson. The regulation of host population growth by parasitic species. Parasitology, 76(02):119$157,1978$.

[2] R. M. Anderson and R. M. May. The population dynamics of microparasites and their invertebrate hosts. Philosophical Transactions of the Royal Society of London B: Biological Sciences, 291(1054):451-524, 1981.

[3] Laurence E. Atkins. The Hive and the Honey Bee. Dadant \& Sons, Hamilton, IL, 1975.

[4] Larissa L. Bailey and Brenda V. Ball. Honey bee pathology. Academic Press Limited, second edition, 1991.

[5] Roy J. Barker and Yolanda Lehner. Acceptance and sustenance value of naturally occurring sugars fed to newly emerged adult workers of honey bees (Apis mellifera L.). Journal of Experimental Zoology, 187(2):277-285, 1974.

[6] M. A. Becher, J. L. Osborne, P. Thorbek, P. J. Kennedy, and V. Grimm. Towards a systems approach for understanding honeybee decline: a stocktaking and synthesis of existing models. Journal of Applied Ecology, 50:868-880, 2013.

[7] Ric Bessin. Varroa mites infesting honey bee colonies. http://www2 . ca.uky . edu/entomology/entfacts/ef608.asp, 2013.

[8] Matt I. Betti, Lindi M. Wahl, and Mair Zamir. Effects of infection on honey bee population dynamics: A model. PloS one, 9(10):e110237, 2014.

[9] Rafael A. Calderón, Luis G. Zamora, Johan W. Van Veen, and Mariela V. Quesada. A comparison of the reproductive ability of Varroa destructor (Mesostigmata: Varroidae) in worker and drone brood of Africanized honey bees (Apis mellifera). Experimental and Applied Acarology, 43(1):25-32, 2007.

[10] Carlos Castillo-Chavez and Horst R. Thieme. Asymptotically autonomous epidemic models. In Proceeding Third International Conference on Mathematical Population Dynamics, (Ovide Arino and Marek Kimmel Eds.), pages 33-50. Wuerz, Winnipeg, Canada, 1995.

[11] Yanping Chen, Jay Evans, and Mark Feldlaufer. Horizontal and vertical transmission of viruses in the honey bee, Apis mellifera. Journal of Invertebrate Pathology, 92(3):152-159, 2006.

[12] Yanping Chen, Jeffery S. Pettis, Anita Collins, and Mark F. Feldlaufer. Prevalence and transmission of honeybee viruses. Applied and Environmental Microbiology, 72(1):606-611, 2006.

[13] Diana L. Cox-Foster, Sean Conlan, Edward C. Holmes, Gustavo Palacios, Jay D. Evans, Nancy A. Moran, Phenix-Lan Quan, Thomas Briese, Mady Hornig, David M. Geiser, Vince Martinson, Dennis vanEngelsdorp, Abby L. Kalkstein, Andrew Drysdale, Jeffrey Hui, Junhui Zhai, Liwang Cui, Stephen K. Hutchison, Jan Fredrik Simons, Michael Egholm, Jeffery S. Pettis, and W. Ian Lipkin. A metagenomic survey of microbes in honey bee colony collapse disorder. Science, 318(5848):283-287, 2007.

[14] Gloria DeGrandi-Hoffman, Yanping Chen, Eden Huang, and Ming Hua Huang. The effect of diet on protein concentration, hypopharyngeal gland development and virus load in worker honey bees (Apis mellifera L.). Journal of Insect Physiology, 56(9):1184-1191, 2010.

[15] Gloria DeGrandi-Hoffman and Robert Curry. A mathematical model of Varroa mite (Varroa destructor anderson and trueman) and honeybee (Apis mellifera 1.) population dynamics. International Journal of Acarology, 30(3):259-274, 2004. 
[16] Gloria DeGrandi-Hoffman, Stephen A. Roth, Gerald M. Loper, and Eric H. Erickson. BEEPOP: A honeybee population dynamics simulation model. Ecological Modelling, 45(2):133-150, 1989.

[17] Garance Di Pasquale, Marion Salignon, Yves Le Conte, Luc P. Belzunces, Axel Decourtye, André Kretzschmar, Séverine Suchail, Jean-Luc Brunet, and Cédric Alaux. Influence of pollen nutrition on honey bee health: Do pollen quality and diversity matter? PloS One, 8(8):e72016, 2013.

[18] Hermann J. Eberl, Mallory R. Frederick, and Peter G. Kevan. Importance of brood maintenance terms in simple models of the honeybee - varroa destructor - acute bee paralysis virus complex. In Electronic Journal of Differential Equations, volume 19, pages 85-98, 2010.

[19] Frank A. Eischen and R. Henry Graham. Feeding overwintering honey bee colonies infected with Nosema ceranae. American Bee Journal, 148:555-556, 2008.

[20] James D. Ellis, Jay D. Evans, and Jeffery S. Pettis. Colony losses, managed colony population decline, and colony collapse disorder in the united states. Journal of Apicultural Research, 49(1):134-136, 2010.

[21] James D. Ellis and C. M. Zettel Nalen. Varroa mite, Varroa destructor Anderson and Trueman (Arachnida: Acari: Varroidae). EENY-473. Entomology and Nematology Department, Florida Cooperative Extension Service, Institute of Food and Agricultural Sciences, University of Florida. EEUU., 2010.

[22] E. Frey and P. Rosenkrantz. Autumn invasion rates of varroa destructor (mesostigmata: Varroidae) into honey bee (hymenoptera: Apidae) colonies and the resulting increase in mite populations. J. Econ. Entomol., 107:508-515, 2014.

[23] R. M. Goodwin, M. A. Taylor, H. M. McBrydie, and H. M. Cox. Drift of varroa destructor infested worker honeybees to neighbouring colonies. J. Apic. Res., 45:155-156, 2006.

[24] M. Greatti, N. Milani, and F. Nazzi. Reinfestation of an acaricide -treated apiary by varroa jacobsoni oud. Exp. Appl. Acarol., 16:279-286, 1992.

[25] Ernesto Guzmán-Novoa, Leslie Eccles, Yireli Calvete, Janine Mcgowan, Paul G Kelly, and Adriana Correa-Benítez. Varroa destructor is the main culprit for the death and reduced populations of overwintered honey bee (Apis mellifera) colonies in Ontario, Canada. Apidologie, 41(4):443-450, 2010.

[26] A. C. Highland, A. El Nagar, L. C. M. Mackinder, L. M. L. J. Noel, S. J. Hall, M. J. abd Martin, and D. C. Schroeder. Deformed wing virus implicated in overwintering honeybee colony losses. Appl. Environ. Microbiol., 75:7212-7220, 2009.

[27] Dariel E. Howell and Robert L. Usinger. Observations on the flight and length of life of drone bees. Annals of the Entomological Society of America, 26(2):239-246, 1933.

[28] J. Iqbal and U. Mueller. Virus infection causes specific learning deficits in honeybee foragers. Proc. $R$. Soc B., 274:1517-1521, 2007.

[29] Renée Johnson. Honey Bee Colony Collapse Disorder. CRS report for Congressional Research Service Washington, Washington, D.C., 2010.

[30] Yun Kang, Rebecca Clark, Michael Makiyama, and Jennifer Fewell. Mathematical modeling on obligate mutualism: Interactions between leaf-cutter ants and their fungus garden. Journal of Theoretical Biology, 289:116-127, 2011.

[31] David S. Khoury, Andrew B. Barron, and Mary R. Myerscough. Modelling food and population dynamics in honey bee colonies. PloS One, 8(5):e59084, 2013.

[32] David S. Khoury, Mary R. Myerscough, and Andrew B. Barron. A quantitative model of honey bee colony population dynamics. PloS One, 6(4):e18491, 2011. 
[33] J. Kralj and S. Fuchs. Parasitic varroa destructor mites influence flight duration and homing ability of infested apis mellifera foragers. Apidologie, 37:577-587, 2006.

[34] Christopher M Kribs-Zaleta and Christopher Mitchell. Modeling colony collapse disorder in honeybees as a contagion. Mathematical Biosciences and Engineering, 11(6):1275-1294, 2014.

[35] Yves Le Conte, Marion Ellis, and Wolfgang Ritter. Varroa mites and honey bee health: can Varroa explain part of the colony losses? Apidologie, 41(3):353-363, 2010.

[36] Z. Li, Y. Chen, S. Zhang, S. Chen, W. Li, and et al. Viral infection affects sucrose responsiveness and homing ability of forager honey bees, apis mellifera l. PLoS ONE, 8(10):e77354, 2013.

[37] Zhien Ma, Yicang Zhou, and Jianhong Wu. Modeling and Dynamics of Infectious diseases. Higher Education Press, Beijing and World Scientific Press, Singapore, 2009.

[38] Rob Manning, April Rutkay, Linda Eaton, and Bernard Dell. Lipid-enhanced pollen and lipid-reduced flour diets and their effect on the longevity of honey bees (Apis mellifera L.). Australian Journal of Entomology, 46(3):251-257, 2007.

[39] Stephen J. Martin. Ontogenesis of the mite Varroa jacobson Oud. in worker brood of the honeybee Apis mellifera L. under natural conditions. Experimental \& Applied Acarology, 18(2):87-100, 1994.

[40] Stephen J. Martin. A population model for the ectoparasitic mite Varroa jacobsoni in honey bee (Apis mellifera) colonies. Ecological Modelling, 109(3):267-281, 1998.

[41] Stephen J. Martin. The role of Varroa and viral pathogens in the collapse of honeybee colonies: a modelling approach. Journal of Applied Ecology, 38(5):1082-1093, 2001.

[42] Anna Maurizio. Pollenernährung und lebensvorgänge bei der honigbiene (apis mellifica 1.). Landwirtschaftliches Jahrbuch der Schweiz, 68:115-182, 1954.

[43] Fanny Mondet, Joachim R. de Miranda, Andre Kretzschmar, Yves Le Conte, and Alison R. Mercer. On the front line: Quantitative virus dynamics in honeybee (Apis mellifera L.) colonies along a new expansion front of the parasite Varroa destructor. PLoS Pathogens, 10(8):e1004323, 2014.

[44] Robin F.A. Moritz, F Bernhard Kraus, Per Kryger, and Robin M. Crewe. The size of wild honeybee populations (Apis mellifera) and its implications for the conservation of honeybees. Journal of Insect Conservation, 11(4):391-397, 2007.

[45] F. Nazzi and F. Pennacchio. Disentangling multipleinteractions in the hive ecosystem. Trends in Parisitol, 30:556-561, 2014.

[46] Benjamin P. Oldroyd. What's killing American honey bees? PLoS Biology, 5(6):e168, 2007.

[47] Robert E. Page and Christine Y.S. Peng. Aging and development in social insects with emphasis on the honey bee, Apis mellifera L. Experimental Gerontology, 36(4):695-711, 2001.

[48] Ying-Shin Peng, Yuenzhen Fang, Shaoyu Xu, and Lisheng Ge. The resistance mechanism of the Asian honey bee, Apis cerana Fabr., to an ectoparasitic mite, Varroa jacobsoni Oudemans. Journal of Invertebrate Pathology, 49(1):54-60, 1987.

[49] Lawrence Perko. Differential Equations and Dynamical Systems, volume 7. Springer Science \& Business Media, 2001.

[50] Clint J. Perry, Eirik Søvik, Mary R. Myerscough, and Andrew B. Barron. Rapid behavioral maturation accelerates failure of stressed honey bee colonies. Proceedings of the National Academy of Sciences, 112(11):3427-3432, 2015. 
51] V. Ratti, P. G. Kevan, and H. J. Eberl. A mathematical model of the honeybee-varroa destructor-acute bee paralysis virus system with seasonal effects. Bulletin of Mathematical Biology, 77(8):1493-1520, 2015.

[52] Vardayani Ratti, Peter G. Kevan, and Hermann J. Eberl. A mathematical model for population dynamics in honeybee colonies infested with Varroa destructor and the acute bee paralysis virus. Canadian Applied Mathematics Quarterly: accepted, 2012.

[53] Thomas E. Rinderer and Kathleen D. Elliott. Worker honey bee response to infection with Nosema apis: Influence of diet. Journal of Economic Entomology, 70(4):431-433, 1977.

[54] P. Rosenkranz, P. Aumeier, and Ziegelmann B. Biology and control of varroa destructor. J Invertebr Pathol., 103:96-119, 2010.

[55] Stephen Russell, Andrew B. Barronb, and David Harris. Dynamic modelling of honey bee (apis mellifera) colony growth and failure. Ecological Modelling, 265(10):158-169, 2013.

[56] F. Sakofski, N Koeniger, and S. Fuchs. Disentangling multipleinteractions in the hive ecosystem. Apidologie, 21:547-550, 1990.

[57] Diana Sammataro, Uri Gerson, and Glen Needham. Parasitic mites of honey bees: life history, implications, and impact. Annual Review of Entomology, 45(1):519-548, 2000.

[58] Thomas Schmickl and Karl Crailsheim. Inner nest homeostasis in a changing environment with special emphasis on honey bee brood nursing and pollen supply. Apidologie, 35(3):249-263, 2004.

[59] Thomas Schmickl and Karl Crailsheim. HoPoMo: A model of honeybee intracolonial population dynamics and resource management. Ecological Modelling, 204(1):219-245, 2007.

[60] Justin O. Schmidt, Steven C. Thoenes, and Marshall D. Levin. Survival of honey bees, apis mellifera (hymenoptera: Apidae), fed various pollen sources. Annals of the Entomological Society of America, 80(2):176-183, 1987.

[61] Li S. Schmidt, Justin O. Schmidt, Hima Rao, Weiyi Wang, and Ligen Xu. Feeding preference and survival of young worker honey bees (Hymenoptera: Apidae) fed rape, sesame, and sunflower pollen. Journal of Economic Entomology, 88(6):1591-1595, 1995.

[62] Thomas D. Seeley. Life history strategy of the honey bee, Apis mellifera. Oecologia, 32(1):109-118, 1978.

[63] Miaoqing Shen, Liwang Cui, Nancy Ostiguy, and Diana L. Cox-Foster. Intricate transmission routes and interactions between picorna-like viruses (Kashmir bee virus and sacbrood virus) with the honeybee host and the parasitic varroa mite. Journal of General Virology, 86(8):2281-2289, 2005.

[64] Niko Speybroeck, Jay D. Evans, Bach Kim Nguyen, Chris Mullin, Maryann Frazier, Jim Frazier, Diana Cox-Foster, Yanping Chen, David R Tarpy, Eric Haubruge, Chris Mullin, Maryann Frazier, Jim Frazier, Diana L. Cox-Foster, Yanping Chen, David R. Tarpy, Eric Haubruge, Jeffery S. Pettis, and Claude Saegerman. Weighing risk factors associated with bee colony collapse disorder by classification and regression tree analysis. Journal of Economic Entomology, 103(5):1517-1523, 2010.

[65] Nathalie A. Steinhauer, Karen Rennich, Michael E. Wilson, Dewey M. Caron, Eugene J. Lengerich, Jeffery S. Pettis, Rose Robyn, John A. Skinner, David R. Tarpy, James T. Wilkes, and Dennis vanEngelsdorp. A national survey of managed honey bee 2012-2013 annual colony losses in the USA: results from the Bee Informed Partnership. Journal of Apicultural Research, 53(1):1-18, 2014.

[66] David J.T. Sumpter and Stephen J. Martin. The dynamics of virus epidemics in Varroa-infested honey bee colonies. Journal of Animal Ecology, 73(1):51-63, 2004. 
[67] Horst R. Thieme, Thanate Dhirasakdanon, Zhun Han, and Roy Trevino. Species decline and extinction: synergy of infectious disease and Allee effect? Journal of Biological Dynamics, 3(2-3):305-323, 2009.

[68] Dennis VanEngelsdorp, Jay D. Evans, Claude Saegerman, Chris Mullin, Eric Haubruge, Bach Kim Nguyen, Maryann Frazier, Jim Frazier, Diana L. Cox-Foster, Yanping Chen, Robyn Underwood, David R. Tarpy, and Jeffery S. Pettis. Colony collapse disorder: A descriptive study. PloS one, 4(8):e6481, 2009.

[69] Dennis vanEngelsdorp and Marina Doris Meixner. A historical review of managed honey bee populations in Europe and the United States and the factors that may affect them. Journal of Invertebrate Pathology, 103:S80-S95, 2010.

[70] Kumar Vetharaniam and Nigel D. Barlow. Modelling biocontrol of Varroa destructor using a benign haplotype as a competitive antagonist. New Zealand Journal of Ecology, 30(1):87-102, 2006.

[71] Jinfeng Wang, Junping Shi, and Junjie Wei. Predator-prey system with strong Allee effect in prey. Journal of Mathematical Biology, 62(3):291-331, 2011.

[72] Ying Wang, Osman Kaftanoglu, M. Kim Fondrk, and Robert E. Page. Nurse bee behaviour manipulates worker honeybee (Apis mellifera 1.) reproductive development. Animal Behaviour, 92:253-261, 2014.

[73] Myrna E. Watanabe. Pollination worries rise as honey bees decline. Science, (265):1170, 1994.

[74] David Wilkinson and Graham C. Smith. A model of the mite parasite, Varroa destructor, on honeybees (Apis mellifera) to investigate parameters important to mite population growth. Ecological Modelling, $148(3): 263-275,2002$.

[75] Geoffrey R. Williams, David R. Tarpy, Dennis vanEngelsdorp, Marie-Pierre Chauzat, Diana L. CoxFoster, Keith S. Delaplane, Peter Neumann, Jeffery S. Pettis, Richard E.L. Rogers, and Dave Shutler. Colony collapse disorder in context. Bioessays, 32(10):845-846, 2010. 